\title{
Systematic review of outcome domains and instruments used in designs of clinical trials for interventions that seek to restore bilateral and binaural hearing in adults with unilateral severe to profound sensorineural hearing loss ('single-sided deafness')
}

Roulla Katiri ${ }^{1,2,3} \mathbb{D}$, Deborah A. Hall ${ }^{1,3,4} \mathbb{D}$, Catherine F. Killan ${ }^{1,3,5} \mathbb{D}$, Sandra Smith ${ }^{1,3} \mathbb{D}$, Pattarawadee Prayuenyong ${ }^{1,3,6}$ (D) and Pádraig T. Kitterick ${ }^{1,3,7^{*}}$ (D)

\begin{abstract}
Background: This systematic review aimed to identify, compare and contrast outcome domains and outcome instruments reported in studies investigating interventions that seek to restore bilateral (two-sided) and/or binaural (both ears) hearing in adults with single-sided deafness (SSD). Findings can inform the development of evidencebased guidance to facilitate design decisions for confirmatory trials.

Methods: Records were identified by searching MEDLINE, EMBASE, PubMed, CINAHL, ClinicalTrials.gov, ISRCTN, CENTRAL, WHO ICTRP and the NIHR UK clinical trials gateway. The search included records published from 1946 to March 2020. Included studies were those as follows: (a) recruiting adults aged 18 years or older diagnosed with SSD of average threshold severity worse than $70 \mathrm{~dB} \mathrm{HL}$ in the worse-hearing ear and normal (or near-normal) hearing in the better-hearing ear, (b) evaluating interventions to restore bilateral and/or binaural hearing and (c) enrolling those adults in a controlled trial, before-and-after study or cross-over study. Studies that fell just short of the participant eligibility criteria were included in a separate sensitivity analysis.

(Continued on next page)
\end{abstract}

\footnotetext{
* Correspondence: padraig.kitterick@nottingham.ac.uk

${ }^{1}$ National Institute for Health Research (NIHR) Nottingham Biomedical

Research Centre (BRC), Ropewalk House, 113 The Ropewalk, Nottingham NG1

5DU, UK

${ }^{3}$ Hearing Sciences, Division of Clinical Neuroscience, School of Medicine,

University of Nottingham, Nottingham NG7 2UH, UK

Full list of author information is available at the end of the article
}

C The Author(s). 2021 Open Access This article is licensed under a Creative Commons Attribution 4.0 International License, which permits use, sharing, adaptation, distribution and reproduction in any medium or format, as long as you give appropriate credit to the original author(s) and the source, provide a link to the Creative Commons licence, and indicate if changes were made. The images or other third party material in this article are included in the article's Creative Commons licence, unless indicated otherwise in a credit line to the material. If material is not included in the article's Creative Commons licence and your intended use is not permitted by statutory regulation or exceeds the permitted use, you will need to obtain permission directly from the copyright holder. To view a copy of this licence, visit http://creativecommons.org/licenses/by/4.0/. The Creative Commons Public Domain Dedication waiver (http://creativecommons.org/publicdomain/zero/1.0/) applies to the data made available in this article, unless otherwise stated in a credit line to the data. 
(Continued from previous page)

Results: Ninety-six studies were included (72 full inclusion, 24 sensitivity analysis). For fully included studies, 37 exclusively evaluated interventions to re-establish bilateral hearing and 29 exclusively evaluated interventions to restore binaural hearing. Overall, 520 outcome domains were identified (350 primary and 170 secondary). Speechrelated outcome domains were the most common (74\% of studies), followed by spatial-related domains (60\% of studies). A total of 344 unique outcome instruments were reported. Speech-related outcome domains were measured by 73 different instruments and spatial-related domains by 43 different instruments. There was considerable variability in duration of follow-up, ranging from acute (baseline) testing to 10 years after the intervention. The sensitivity analysis identified no additional outcome domains.

Conclusions: This review identified large variability in the reporting of outcome domains and instruments in studies evaluating the therapeutic benefits and harms of SSD interventions. Reports frequently omitted information on what domains the study intended to assess, and on what instruments were used to measure which domains.

Trial registration: The systematic review protocol is registered on PROSPERO (International Prospective Register of Systematic Reviews): Registration Number CRD42018084274. Registered on 13 March 2018, last revised on 7th of May 2019.

Keywords: Single-sided deafness, Outcome domains, Measurement instruments, Hearing interventions, Clinical trial design

\section{Background}

Single-sided deafness (SSD) arises when there is normal or near-normal hearing in one ear and a severe to profound sensorineural hearing impairment in the other ear [1]. The cause can be congenital [2, 3], sudden (e.g. Meniere's disease [4], idiopathic [5, 6], due to autoimmune systemic diseases [7]); or progressive (e.g. vestibular schwannoma [8-10]). Regardless, SSD can lead to functional [11-16], psychological and social consequences [17-19].

The most commonly used treatments for SSD enable access to sounds on both sides of the head ('bilateral' hearing) by rerouting sounds from the impaired ear to the hearing ear $[20,21]$. This effect can be achieved with a CROS (Contralateral Routing Of Signals) aid [20, 2227] or a bone-anchored hearing aid (BAHA) [28-43] as well as other bone conduction devices like the ADHEAR $[44,45]$ or SoundBite ${ }^{\mathrm{TM}}[34,46-49]$. Alternatively, auditory input to the deaf ear can be restored ('binaural' hearing) by delivering sounds directly to the auditory pathway on the side of the impaired ear using auditory prostheses like a middle ear implant (MEI) $[50,51]$ or a cochlear implant [52-73].

Understanding which of these intervention approaches are optimal for patients with SSD should be based on robust evidence from well-designed trials [74, 75]. Certainly, with respect to health-related quality of life, there is a known inconsistency in the choice of measurement instruments in trials assessing the benefits of SSD interventions [76]. This inconsistency hinders comparison and meta-analysis across studies. The evidence synthesis in systematic reviews for example can be reliably conducted only if trials assess the same outcomes and measure them in the same way [77].
The challenge of synthesising evidence from trials and the importance of utilising valid instruments that effectively measure the intended audiological outcomes has been highlighted by Hall et al. [78]. Trialists should ideally base their choice of outcome measures on what is important and relevant to people making decisions about healthcare [79-81]. The question of what outcome domains are important and relevant to individuals with SSD when deciding whether an intervention works has yet to be asked in a systematic way. One attempt was made in 2017 , but this was based on two discussions among professional experts in cochlear implantation at international conferences [1] and was intended for adoption in clinical practice. There has been no rigorous scrutiny of outcome reporting for rerouting and restoring interventions, no systematic patient involvement and no specific consideration of what should be recommended for clinical trials. As a consequence, investigators adopt markedly different study designs when assessing the clinical benefit of rerouting and restoring interventions for SSD.

The Core Rehabilitation Outcome Set for Single Sided Deafness (CROSSSD) study seeks to examine and address problems with inconsistent outcome reporting in SSD intervention trials. To achieve this, CROSSSD is developing a Core Outcome Set (COS) through a rigorous evidence-based process and by actively involving all relevant stakeholders in decision making. A COS is an agreed minimum set of outcomes or outcome measures which comprises a standardised collection of outcome domains that should be measured and reported worldwide, as a minimum, in all controlled trials within a research area [82-85]. Our published protocol [86] describes the process and this systematic review formed one of the first steps. 


\section{Objectives}

The primary objective of this systematic review was to identify those outcome domains and outcome instruments reported in published clinical studies evaluating rerouting and/or restoring interventions in adults with SSD. This information will be used to subsequently generate a 'long list' of candidate outcomes to be rated by SSD stakeholders according to whether each is important and critical to determine if an intervention works in this clinical population as part of the development of a Core Outcome Set (COS) [86].

There were two additional (secondary) objectives. One was to compare and contrast outcome domains and instruments reported for interventions that aim to reestablish (i) bilateral hearing (i.e. CROS aid, BAHA, ADHEAR, SoundBite ${ }^{\mathrm{m}}$ ) and (ii) binaural hearing (i.e. MEI, CI). The other was to examine what outcome domains had been assessed and outcome instruments used as a function of time point after intervention. This information can be used to distinguish short- and long-term treatment-related changes. An exploratory objective was added to examine how the long list of candidate outcomes would be affected if the audiometric inclusion criteria were more lenient than our working definition adopted from the Van de Heyning et al. [1] consensus paper.

\section{Methods}

\section{Searches}

Details of the specific review questions, search strategy, study eligibility criteria, information sources, selection and data collection processes, quality assessment and data synthesis methods were published on PROSPERO international prospective register of systematic reviews in advance of data extraction [87]. There were no modifications to this PROSPERO protocol, but the Preferred Reporting Items for Systematic reviews and MetaAnalyses (PRISMA) statement [88] was modified for reporting purposes here. A populated PRISMA (2009) checklist can found in Additional file 1.

\section{Study inclusion and exclusion criteria}

The eligibility was defined according to PICOS (Participant, Intervention(s), Comparator(s), Outcome, Setting) criteria. All included records assessed adults (male or female) aged 18 years or older with a diagnosis of congenital or acquired SSD. For the primary objective, diagnoses had to meet an audiometric profile independently defined through consensus [1]. For the worse-hearing ear, this required a threshold severity worse than $70 \mathrm{~dB} \mathrm{HL}$ at audiometric frequencies ranging from 1 to $4 \mathrm{kHz}$, and for the better-hearing ear, this required a pure tone average of $\leq 30 \mathrm{~dB} H \mathrm{HL}$ across the same frequency range.
Eligible interventions comprised any medical devices designed specifically to restore bilateral (two-sided) or binaural (both ears) hearing. Any comparators in the study design were allowed, but studies exclusively evaluating other audiological interventions such as conventional hearing aids, assistive listening devices, audiological counselling, communication strategies or providing no intervention (unaided or placebo) were excluded. There were no restrictions on outcomes or research settings.

The systematic review included records reporting randomised controlled trials, quasi-randomised controlled trials, non-randomised controlled trials, before-and-after studies, cross-over studies, trial registrations and published protocols of such ongoing studies, and systematic reviews. Relevant systematic reviews were not subjected to the data collection process itself but were read to ensure all eligible records had been captured. Case control studies, cohort studies, non-systematic literature reviews (e.g. scoping reviews), practice guidelines, expert opinions, case series, case reports, book chapters, conference papers, manufacturers' articles (e.g. white papers), animal studies and studies that use predictive modelling (e.g. prognostic factors established by acoustic test box measurements or studies performed with cadavers) were excluded. Original searches were performed from 1946, or the start date of databases, whichever was earlier, up to April 2018 inclusive. The searches were updated to 18th of March 2020. There were no restrictions on language of the publication.

During the data collection process, a small number of records were identified where information about agerelated eligibility, audiometric thresholds or type of hearing loss in either the better or poorer hearing ear were missing. The corresponding author was contacted for more details by email, and a decision was made regarding inclusion in light of the new information provided. In cases where the author did not respond, an executive decision was taken to (i) include, (ii) exclude or (iii) use for sensitivity analysis; following discussion with one of the two senior authors (PTK or DAH). Cases in the sensitivity analysis were those trials or studies in which (i) participants' audiometric profiles were close to our adopted SSD definition but differed from those criteria by up to $20 \mathrm{~dB}$ in individual frequencies either in the better or worse ear; (ii) the corresponding author was asked to clarify the audiometric profiles of participants but did not respond; and (iii) ongoing studies recruiting a mixture of participants (including children aged less than 18 years of age) and where it was not clear if results would be reported separately for the adults (aged 18 years or over).

\section{Information sources}

Records were identified by searching electronic databases of research literature including (Table 1). 
Table 1 Table summarising the electronic information sources used and the number of records identified

\begin{tabular}{|c|c|c|c|}
\hline Type of electronic search & Database & Date range & $\begin{array}{l}\text { Number of } \\
\text { items (n) }\end{array}$ \\
\hline \multirow[t]{5}{*}{ Academic databases } & Excerpta Medica dataBASE (EMBASE) via OvidSP & $\begin{array}{l}1974 \text { to } 18 \text { th } \\
\text { March } 2020\end{array}$ & 1463 \\
\hline & Medical Literature Analysis and Retrieval System Online (MEDLINE) via OvidSP & $\begin{array}{l}1974 \text { to } 18 \text { th } \\
\text { March } 2020\end{array}$ & 1144 \\
\hline & PubMed National Centre for Biotechnology Information & $\begin{array}{l}1946 \text { to } 18 \text { th } \\
\text { March } 2020\end{array}$ & 1223 \\
\hline & \multirow[t]{2}{*}{ Cumulative Index of Nursing and Allied Health Literature (CINAHL) via EBSCO } & $\begin{array}{l}1982 \text { to } 18 \text { th } \\
\text { March } 2020\end{array}$ & 384 \\
\hline & & Searched on & \\
\hline \multirow{5}{*}{$\begin{array}{l}\text { Clinical trial registers and/or } \\
\text { other sources }\end{array}$} & ClinicalTrials.gov (www.clinicaltrials.gov) & 18th March 2020 & 193 \\
\hline & $\begin{array}{l}\text { International Standard Randomised Controlled Trials Number (ISRCTN) } \\
\text { Registry (www.isrctn.com) }\end{array}$ & 18th March 2020 & 48 \\
\hline & Cochrane Central Register of Controlled Trials (CENTRAL) & 18th March 2020 & 962 \\
\hline & $\begin{array}{l}\text { World Health Organization (WHO) International Clinical Trials Registry } \\
\text { Platform (ICTRP) (www.who.int/ictrp) }\end{array}$ & 18th March 2020 & 270 \\
\hline & NIHR UK Clinical Trials Gateway (www.ukctg.nihr.ac.uk) & 18th March 2020 & 67 \\
\hline
\end{tabular}

Published, unpublished and ongoing studies were identified by electronically searching the following databases from their inception: EMBASE, MEDLINE, PubMed, CINAHL, ClinicalTrials.gov, ISRCTN, CENTRAL, ICTRP and the NIHR UK Clinical Trials Gateway. Electronic searchers were run by RK and PTK in March and April 2018 and then updated on 18th March 2020. In addition, a hand-search was conducted when reviewing the 76 published articles that had met the eligibility criteria at the abstract and full-text screening stages. Two potential articles were identified [27, 89], but following closer scrutiny neither met eligibility.

\section{Search strategy}

The search strategy used in this systematic review was registered on PROSPERO [87]. Search terms for the PubMed, EMBASE and MEDLINE databases were informed by the PICOS criteria and comprised a set of terms to identify the population combined with a set of terms to identify relevant interventions. Where possible using the database interface, the scope of the search was limited to humans (not animals) and adults (not paediatric). An example of the search syntax for MEDLINE and EMBASE via OvidSP can be found in Additional file 2. The search strategy for the other databases was modelled on this search strategy and adapted where necessary to ensure the strategies were highly sensitive across each of the database interfaces. As an example, the syntax for search of the CENTRAL trials registry of the Cochrane Collaboration can be found in Additional file 3 .

\section{Data management}

RK, DAH and PTK were responsible for data management and maintained editorial rights. All identified records were saved into a Microsoft Excel Masterfile where records were tracked through the screening and data collection process by a unique study identification code.

\section{Selection process}

All records identified by all database searches were uploaded into the EndNote software (Version X7) that was used to remove duplicates using the records' title, list of authors, year of publication and journal of publication. In a few isolated cases, the abstract was also used to double-check if there was duplication, mainly for records that were published in a different language and the translated title or name of journal were different. The resulting number of records were subjected to eligibility screening.

Eligibility screening was carried out by RK, DAH, CFK, SS, PP and PTK, according to the published protocol [87]. For each record, the title and abstract screening decision was captured using a simple set of descriptors: include; unsure possibly include; exclude out of scope; exclude not SSD; exclude not adults; exclude wrong intervention; exclude wrong trial design; incomplete reference; abstract not accessible and sensitivity analysis (Additional file 4). Two co-authors (RK and DAH or PTK) independently performed and/or reviewed each step (i.e. title and abstract screening, full-text screening, data extraction, risk of bias assessment). Records that were included to conduct the sensitivity analysis only were extracted by RK alone. On rare occasions where agreement could not be reached between co-authors, disagreements were resolved by a third reviewer (DAH or PTK). The risk of bias assessment did not affect which findings were included in the analyses. 
To enhance our data quality, data collection was guided by a data extraction protocol (Additional file 5), which informed the headings of the data masterfile. A calibration exercise was conducted for ten included records and reviewed for consistency across two coders, and the data extraction protocol was revised according to the lessons learned. No coder was permitted to be an author on any of the included records.

\section{Data items}

Data items included PICOS fields as described by the PROSPERO record [87]. Participant data items relating to the inclusion criteria for each record were as follows:

(1) SSD cause / aetiology, (2) age range, (3) mean age, (4) age standard deviation and (5) time since SSD diagnosis. Intervention data items recorded were as follows: (6) type of intervention device used and (7) time of implementation of intervention (how long after the onset of SSD the intervention was implemented). Data items describing the trial design included (8) the comparator device (if applicable), (9) the type of trial design and (10) the time duration for which each intervention or comparator device were used.

Outcome data items were as follows: (11) the outcome domain(s) specified by the investigators, (12) instruments specified by the investigators and (13) measurement time frame. Information relating to these three data items was recorded separately for all primary and secondary outcomes. Where authors were not explicit about the distinction between primary and secondary outcomes, the 'Methods' and 'Results' sections of each article were examined to identify any relevant information related to this distinction. If the study investigators did not explicitly distinguish multiple outcome domains as primary or secondary, they were all classified as primary.

Supplementary information was also extracted from each individual record on the following: (1) countries where the study was conducted, (2) corresponding author contact details, (3) source title (e.g. journal), (4) date of publication, (5) primary and/or secondary objective(s), (6) sample size, (7) description of any modifications to the study, particularly any discrepancies between the trial protocol and the subsequent report of the findings, and (8) any conflicts of interest identified by the authors. For (4), the date of publication recorded the date of the print copy, not the date of first submission, acceptance or the date of 'online first' publication. For (6), the estimated sample size was recorded for ongoing clinical trials where enrolment was not yet available. If any information was not reported, then 'not stated' was recorded in the corresponding field.

Where trial records had been consolidated into a single study, the data items reported in the synthesis related to the most recent study publication. For those records in which several pieces of information were consolidated for a single study, any inconsistencies between the protocol and the final reported study findings were noted (e.g. if the intended participant sample size was different in the published clinical trial record in comparison to the final study findings publication).

\section{Outcomes and prioritisation}

The primary research question was to identify those outcome domains and outcome instruments reported in studies investigating interventions that seek to restore hearing in adults with SSD. There are no validated taxonomies specific to the hearing field and so for our classification of outcome domains, we chose to use the Dodd et al. [90] taxonomy. Strengths of this taxonomy are that it has been developed specifically for trial outcomes, is comprehensive, is not disease specific, is not limited to patient-centered outcomes and is applicable to trials irrespective of the field being studied. It comprises 38 categories across five core areas; death, physiological or clinical, life impact, resource use and adverse events. Classification of the review findings with respect to this taxonomy was conducted by RK and PTK. Finer breakdown of outcomes with the category 'Ear and labyrinth outcomes' was informed by a 2-day outcome domain grouping workshop that took place in July 2019 with members of the research steering group and the two public research partners. Details on the individual outcome domains review, consolidation and categorisation during the workshop can be found in the CROSSSD study protocol [86].

\section{Risk of bias}

Given that the primary objective of this systematic review concerns methodology (not therapeutic effects), we limited the assessment of risk of bias to the data collection methods for consolidated records rather than any analysis of the intervention-related changes. The consolidated record data (e.g. outcome descriptors, published primary / secondary findings) was critically analysed for consistency of outcome reporting by two independent reviewers (RK and DAH). Risk of bias was assessed by analysing the reporting of outcomes both within and across manuscripts reporting study findings. Bias was determined by whether outcomes were reported prospectively through trial registration or published protocol, and whether outcomes were reported consistently between protocol / registration and study report. If consensus could not be reached on whether outcomes had been reported consistently, then disagreements were resolved by discussion with a third reviewer (PTK). No contact was made with corresponding authors to investigate the rationale of altered reporting. The quality of a 
record did not affect its inclusion in the synthesis of outcomes.

\section{Results}

\section{Search results}

Figure 1 displays the results of the search strategy used to identify the relevant articles as recommended by the PRISMA statement [91]. The search strategy yielded 5754 records from which 2554 were excluded as duplicates. This resulted in a total of 3200 unique records being subjected to eligibility screening. Most exclusions during title and abstract eligibility screening were due to the study being out of scope (e.g. examined surgical methods, assistive hearing devices or hearing therapy techniques), the wrong trial design (e.g. case series, scoping reviews), not recruiting SSD participants or not recruiting adults, leaving 564 records (Fig. 1). For these, full texts were obtained and where necessary were translated to the English language. Full-text eligibility screening enabled a further 446 records to be excluded, with most exclusions due to participants not meeting the working definition of SSD [1] $(n=281)$ or using an ineligible trial design $(n=111)$. These exclusions left 118 records for data extraction, detailed as follows.

CROSSSD Study Systematic Review PRISMA Flow Diagram

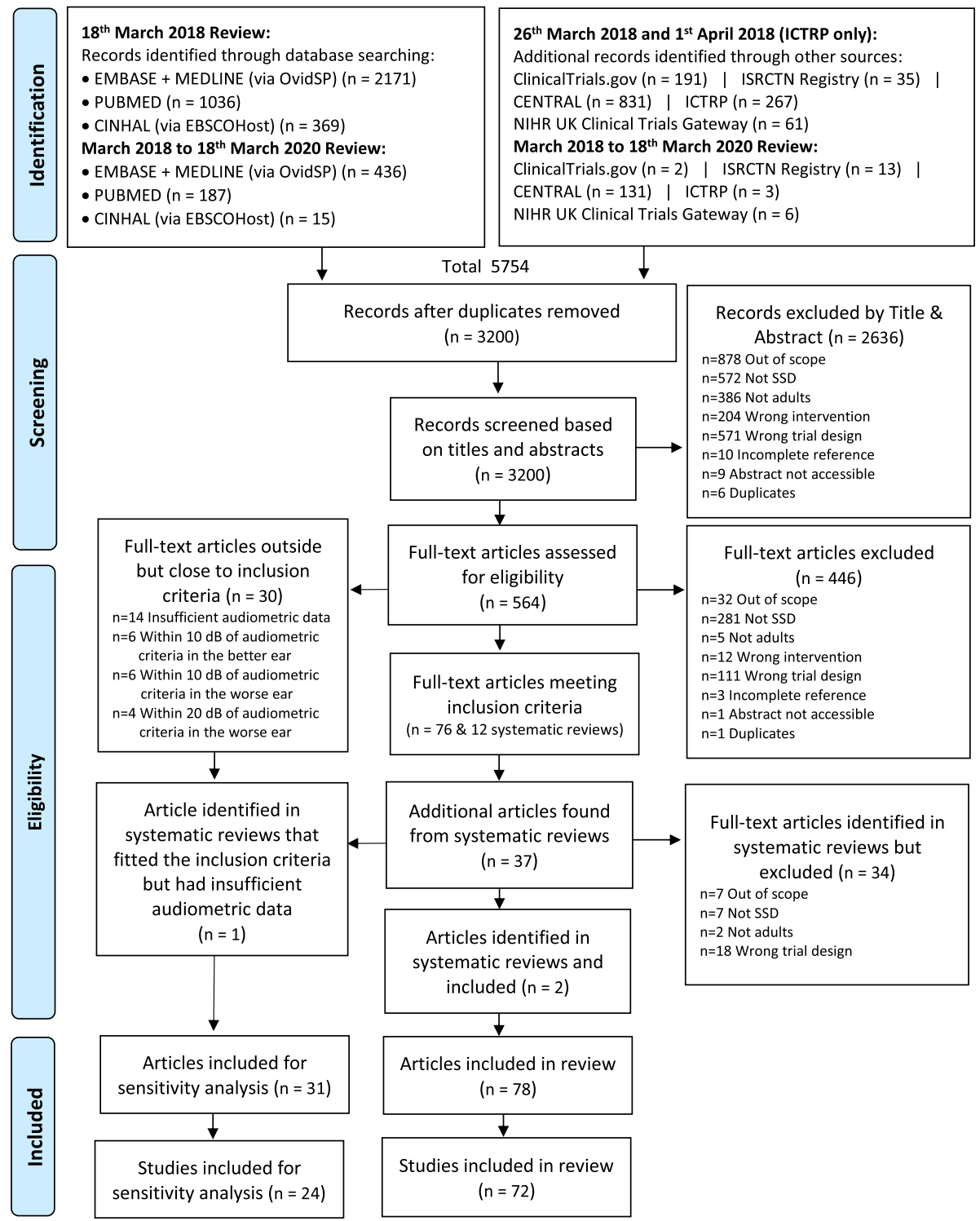

Fig. 1 CROSSSD Reporting Items for Systematic Reviews and Meta-Analyses (PRISMA) flow diagram [91] 
Full-text screening confirmed that 76 records reported trials in which the diagnosis of SSD fully met our criteria according to the Van de Heyning et al. [1] definition. The remaining 30 records reported participant criteria that narrowly missed the inclusion criteria (see eligibility criteria section above for details) but were sufficiently close to the criteria for inclusion in the sensitivity analysis. The 12 systematic review articles [76, 92-102] identified by the searches were reviewed to check for any overlooked trials or studies for inclusion, and 37 additional articles were identified by this approach. Fulltext screening established that two met the inclusion criteria [72, 73], and one was included in the sensitivity analysis [25]. The rest were excluded due to wrong trial design $(n=18)$, the majority were conference papers, or they were out of scope $(n=7)$ [103-109], did not fit the audiometric criteria $(n=7)$ [110-116], or included children in their studies that were not reported separately from adults $(n=2)$ [117, 118]. References for the included records are given in Additional file 6 and the data masterfile containing information on all the data items is available for download (Additional file 7).

A number of records were consolidated for the purpose of reporting because they described the same trial or study. Two records by Härkönen et al. [53, 54] reported different measures obtained from the same group of participants. Two records reporting on a United Sates of America (USA) multicentre study [31, 32] were also consolidated as they reported on the same subset of participants. Four records [46-48, 119] reported on the same USA trial and participants, but presented different outcomes at different time scales so they were grouped. A French clinical trial registration (NCT02204618) by Marx et al. [120] was consolidated with the study findings record [69]. Similarly, a Swiss clinical trial registration (NCT01749592) [121] was consolidated with the published study findings record [66], a USA clinical trial (NCT02259192) [122] with the equivalent published record [65] and another French clinical trial (NCT02966366) [123] with its published record [67]. Two records by the Arndt et al. group reported on the same 11 participants but presented outcomes at 6 months [59] and at 12 months [52], so they were consolidated. Conversely, one composite article [38] reported the methods and results of five separate trials. Data from this article were extracted as five distinct studies. This re-classification led to a final dataset of 78 records reporting 72 studies that met full eligibility, and 31 records reporting 24 studies for the sensitivity analysis (Fig. 1). Of the 72 studies included, 37 assessed rerouting interventions, 29 studies assessed restoring interventions and just 6 studies directly evaluated both types of interventions.

\section{Data collection process}

During eligibility screening, corresponding authors for 17 records were contacted to ask for more detail on the participant audiometric eligibility criteria or the definition of SSD adopted (see Additional file 8). Six authors responded with new information that allowed the screeners to come to a decision to (i) include for data extraction and synthesis $(n=1)$ [124], (ii) exclude from data extraction $(n=2)[125,126]$ or (iii) include for sensitivity analysis $(n=3)[123,127,128]$. Three emails were undeliverable, for these we decided to exclude two records $[129,130]$ but include one record [131] which was a clinical trial intending to recruit participants with SSD. Six authors did not respond [99, 132-136], and so we decided to include all six for sensitivity analysis. Two authors responded but their responses did not adequately clarify the query; one record [137] was included for sensitivity analysis and one [138] was excluded from data extraction.

The most common SSD diagnoses were sudden idiopathic or unknown cause $(n=218,41 \%)$ and vestibular schwannoma $(n=134,25 \%)$. The majority of rerouting intervention studies were conducted in the USA $(n=25$, $66 \%)$. Restoring intervention studies were conducted in the USA $(n=8,36 \%)$, Belgium and Germany $(n=4$, $19 \%)$. Studies recruited a median of 10.5 participants $($ mean $=25.3$, range 3-160). Most multicentre studies $(n=7)$ were conducted to evaluate restoring interventions rather than rerouting interventions.

\section{Outcome domains}

To address our first objective, we examined the outcome domains extracted data from the 72 included studies and classified them for reporting using the Dodd et al. [90] taxonomy. Overall, 350 primary and 170 secondary data items were categorised across 19 of the 38 taxonomy categories (Table 2). Just over half $(55 \%)$ of the reported outcome domains were physiological or clinical outcomes in the ear and labyrinth category (194 primary and 90 secondary outcome domain data items). Within this category, the most common items were from speech-related domains (e.g. speech in noise and speech in quiet), spatial-related domains (e.g. localisation and spatial hearing), hearing thresholds and tinnitus loudness. Life impact was the next most frequently reported core area $(33 \% ; 120$ primary and 50 secondary outcome domain data items). The most popular categories within life impact were delivery of care and quality of life. We observed that investigators sometimes reported multiple assessments of the same outcome domain within a study, and so the caveat to these findings is that these frequencies do somewhat over-estimate the proportion of included studies reporting the outcome domain. A complete list of all reported outcome domains can be 
Table 2 Summary of primary and secondary outcome domains and data items across all 72 included studies, classified according to core areas and categories defined by Dodd et al. [90]. Percentage values less than $1 \%$ are not reported

\begin{tabular}{|c|c|c|c|c|c|c|c|}
\hline $\begin{array}{l}\text { Taxonomy } \\
\text { core area }\end{array}$ & $\begin{array}{l}\text { Taxonomy } \\
\text { categories }\end{array}$ & $\begin{array}{l}\text { Number of } \\
\text { data items } \\
\text { (primary } \\
\text { outcome } \\
\text { domains) } \\
\end{array}$ & $\begin{array}{l}\% \text { of total } \\
\text { number of } \\
\text { primary } \\
\text { outcome } \\
\text { domains } \\
\end{array}$ & $\begin{array}{l}\text { Number of data } \\
\text { items } \\
\text { (secondary } \\
\text { outcome } \\
\text { domains) }\end{array}$ & $\begin{array}{l}\% \text { of total } \\
\text { number of } \\
\text { secondary } \\
\text { outcome } \\
\text { domains } \\
\end{array}$ & $\begin{array}{l}\text { Number of } \\
\text { unique outcome } \\
\text { domains } \\
\text { reported as } \\
\text { primary }\end{array}$ & $\begin{array}{l}\text { Number of unique } \\
\text { outcome domains } \\
\text { reported as } \\
\text { secondary only }\end{array}$ \\
\hline Death & $\begin{array}{l}\text { 1: Mortality / } \\
\text { survival }\end{array}$ & 0 & - & 0 & - & 0 & 0 \\
\hline \multirow[t]{6}{*}{$\begin{array}{l}\text { Physiological } \\
\text { or clinical }\end{array}$} & $\begin{array}{l}\text { 6: Ear and } \\
\text { labyrinth } \\
\text { outcomes }\end{array}$ & 194 & $55.4 \%$ & 90 & $52.9 \%$ & 15 & 3 \\
\hline & $\begin{array}{l}\text { 7: Eye } \\
\text { outcomes }\end{array}$ & 0 & - & 1 & - & 1 & 0 \\
\hline & $\begin{array}{l}\text { 9: General } \\
\text { outcomes }\end{array}$ & 9 & $2.6 \%$ & 1 & - & 2 & 0 \\
\hline & $\begin{array}{l}\text { 17: Nervous } \\
\text { system } \\
\text { outcomes }\end{array}$ & 3 & - & 3 & $1.8 \%$ & 2 & 0 \\
\hline & $\begin{array}{l}\text { 21: Psychiatric } \\
\text { outcomes }\end{array}$ & 3 & - & 0 & - & 1 & 0 \\
\hline & $\begin{array}{l}\text { 23: Skin and } \\
\text { subcutaneous } \\
\text { tissue outcomes }\end{array}$ & 2 & - & 0 & - & 1 & 0 \\
\hline \multirow[t]{9}{*}{ Life impact } & $\begin{array}{l}\text { 25: Physical } \\
\text { functioning }\end{array}$ & 2 & - & 5 & $3.3 \%$ & 2 & 3 \\
\hline & $\begin{array}{l}\text { 26: Social } \\
\text { functioning }\end{array}$ & 1 & - & 3 & $2.9 \%$ & 1 & 2 \\
\hline & $\begin{array}{l}\text { 27: Role } \\
\text { functioning }\end{array}$ & 1 & - & 1 & - & 1 & 0 \\
\hline & $\begin{array}{l}\text { 28: Emotional } \\
\text { functioning / } \\
\text { well-being }\end{array}$ & 7 & $2.0 \%$ & 5 & $3.3 \%$ & 5 & 3 \\
\hline & $\begin{array}{l}\text { 29: Cognitive } \\
\text { functioning }\end{array}$ & 2 & - & 3 & $2.9 \%$ & 1 & 2 \\
\hline & $\begin{array}{l}\text { 30: Global } \\
\text { quality of life }\end{array}$ & 55 & $15.7 \%$ & 16 & $9.4 \%$ & 7 & 0 \\
\hline & $\begin{array}{l}\text { 31: Perceived } \\
\text { health status }\end{array}$ & 1 & - & - & - & 1 & 0 \\
\hline & $\begin{array}{l}\text { 32a: Delivery of } \\
\text { care- } \\
\text { satisfaction / } \\
\text { patient } \\
\text { preference }\end{array}$ & 51 & $14.6 \%$ & 17 & $10.0 \%$ & 10 & 2 \\
\hline & $\begin{array}{l}\text { 32b: Delivery of } \\
\text { care- } \\
\text { acceptability } \\
\text { and availability }\end{array}$ & 0 & - & 1 & - & 0 & 1 \\
\hline \multirow[t]{3}{*}{ Resource use } & 34: Economic & 2 & - & 2 & $1.2 \%$ & 2 & 0 \\
\hline & 35: Hospital & 1 & - & 0 & - & 1 & 0 \\
\hline & $\begin{array}{l}\text { 37: Societal / } \\
\text { carer burden }\end{array}$ & 0 & - & 1 & - & 0 & 1 \\
\hline $\begin{array}{l}\text { Adverse } \\
\text { events }\end{array}$ & $\begin{array}{l}\text { 38: Adverse } \\
\text { events / effects }\end{array}$ & 11 & $3.1 \%$ & 5 & $2.9 \%$ & 3 & 0 \\
\hline \multirow[t]{2}{*}{ Cannot code } & 0: Cannot code & 5 & $1.4 \%$ & 17 & $10.0 \%$ & N/A & N/A \\
\hline & Total & 350 & & 170 & & 55 & 18 \\
\hline
\end{tabular}


found in Additional file 9. A total of 22 outcome domain data items (4\%) could not be coded because they were not clearly defined by the authors. Overall, 73 unique outcome domains were reported across the 72 included studies, 55 primary and 18 secondary outcome domains (Table 2).

\section{Outcome instruments}

Our first objective also asked about the outcome instruments used to measure the domains. For reporting purposes, measurement instruments are summarised according to whether they were as follows: (1) investigator administered, (2) patient-reported outcome measures (PROMs) and (3) unclear or unknown (Table 3). Within each of these categories, a finer breakdown was performed that was relevant to the instrument category (e.g. PROMs could be a numerical rating scale, multi-item questionnaire or diary).

Collating information about the measurement instruments reported in the 72 studies revealed a large number of ways to measure the domains of interest and the fact that no single instrument was used by all studies. We observed that reporting was strongly biased towards benefits not harms. Counting the exact number of instruments is not straightforward because some of the instruments were reported both as global scores and subscale scores across different studies and different authors administered the same instrument to assess different outcome domains. For example, the Glasgow Hearing Aid Benefit Profile (GHABP) [139] was reported in various forms under hearing handicap, preintervention disability, device benefit, device use, satisfaction, and residual (aided) disability. Regarding the Abbreviated Profile of Hearing Aid Benefit (APHAB) [140], performance was most often reported as a subscale not a global score, including all three speech communication subscales (Ease of communication, Reverberation and Background noise subscales). For the purposes of reporting here, the different forms reported by the authors all contribute to the data item counts and so the numbers may over-estimate the number of instruments per se. For that reason, we refer to these data as measurement 'methods' not 'instruments'. A summary of the number of methods used to measure the outcomes across the most popular Dodd's taxonomy categories is given in Table 3. For transparency, a comprehensive listing of all methods can be found in Additional file 10, organised according to the domains within Dodd's taxonomy categories.

Considering the ear and labyrinth outcome domains, the 18 outcome domains were assessed by 133 different measurement methods. A description of the more popular methods is given in Table 4. The most common approach was an investigator-administered psychophysical instrument. This was true for all of the speech-related domains (i.e. speech in noise, speech in quiet and speech hearing), spatial localisation and hearing thresholds. Speech performance was most often measured by a speech reception threshold, although there were many different testing methods. There was no clear preferred method for measuring speech in quiet, while speech in noise was most often assessed using the Hearing in Noise Test (HINT) [141]. However, even here the choice of background noise was not consistent across studies. Localisation performance was most often measured by localisation accuracy using a horizontal circular or semicircular array of loudspeakers. However, again the number of loudspeakers and angular separation between sound sources varied across studies. Perhaps unsurprisingly, hearing thresholds were most often assessed using pure-tone audiometry which tends to have a more standardised testing method. Tinnitus loudness was commonly measured using a visual analogue scale (VAS), which is a form of PROM.

The seven global quality of life outcome domains were assessed by 36 different measurement methods. A description of the more popular methods is given in Table 5. The most common method of assessment was a PROM, in the form of a multi-item questionnaire. Most frequently reported were the Speech, Spatial and Qualities of Hearing (SSQ) [16], Glasgow Benefit Inventory (GBI), single-sided deafness (SSD) questionnaire and Tinnitus Handicap Inventory (THI) [142].

The 12 delivery of care (Satisfaction / patient preference) outcome domains were assessed by 37 different measurement methods. A description of the more popular methods is given in Table 6. Once again, the most common method of assessment was a PROM, in the form of a multi-item questionnaire. The most frequently reported were the Abbreviated Profile of Hearing Aid Benefit (APHAB) [140] and the Glasgow Hearing Aid Benefit Profile (GHABP).

\section{Comparing the report of outcome domains and instruments across interventions}

One of our secondary objectives was to compare and contrast outcome domains and instruments reported for interventions that aim to re-establish (i) bilateral hearing (i.e. CROS aid, BAHA, ADHEAR, SoundBite ${ }^{\mathrm{m}}$ ) through rerouting and (ii) binaural hearing (i.e. MEI, CI) through restoring.

Across the 72 included studies, 37 assessed rerouting interventions only and 29 assessed restoring interventions only. The remainder assessed both interventions in the same study design and so are not included in this comparison. Generally speaking, the two intervention approaches assessed the same outcome domains. But there were several notable exceptions. Tinnitus-related outcomes were almost exclusively limited to studies 
Table 3 Summary of the number of more common measurement methods used to assess treatment outcomes in each domain category from the Dodd et al. [90] taxonomy. Only items where there were more than 10 reports of the outcome domain (Additional file 9) and only those methods reported more than once across the 72 included studies are selected for reporting here. See Additional file 10 for a complete list of instruments

\begin{tabular}{|c|c|c|c|c|c|c|c|c|}
\hline \multirow[t]{2}{*}{$\begin{array}{l}\text { Outcome } \\
\text { domains }\end{array}$} & \multicolumn{4}{|c|}{ Investigator administered } & \multicolumn{3}{|c|}{$\begin{array}{l}\text { Patient-reported outcome measures } \\
\text { (PROMs) }\end{array}$} & \multirow{2}{*}{$\begin{array}{l}\text { Unclear } \\
\text { Unclear }\end{array}$} \\
\hline & $\begin{array}{l}\text { Psychophysical } \\
\text { instruments }\end{array}$ & $\begin{array}{l}\text { Objective } \\
\text { instruments }\end{array}$ & $\begin{array}{l}\text { Technical and } \\
\text { lab measures }\end{array}$ & $\begin{array}{l}\text { Investigator } \\
\text { observation / } \\
\text { judgement }\end{array}$ & $\begin{array}{l}\text { Numerical } \\
\text { rating scale }\end{array}$ & $\begin{array}{l}\text { Multi-item } \\
\text { questionnaire }\end{array}$ & Diary & \\
\hline \multicolumn{9}{|c|}{ Physiological or clinical core area, 6: ear and labyrinth outcomes } \\
\hline $\begin{array}{l}\text { Hearing } \\
\text { thresholds }\end{array}$ & 8 & 1 & - & - & - & - & - & - \\
\hline Speech in noise & 47 & - & - & - & - & 2 & - & - \\
\hline Speech in quiet & 18 & - & - & - & - & 2 & - & - \\
\hline Speech hearing & 6 & - & - & - & - & 3 & - & - \\
\hline $\begin{array}{l}\text { Tinnitus } \\
\text { loudness }\end{array}$ & 1 & - & - & - & 2 & - & - & - \\
\hline Spatial hearing & 1 & - & - & - & - & 3 & - & - \\
\hline Localisation & 33 & - & - & - & - & 1 & - & - \\
\hline $\begin{array}{l}\text { Quality of } \\
\text { hearing }\end{array}$ & - & - & - & - & 2 & 1 & - & 2 \\
\hline Reverberation & - & - & - & - & 1 & 1 & - & - \\
\hline \multicolumn{9}{|c|}{ Life impact core area, 30: global quality of life } \\
\hline $\begin{array}{l}\text { Tinnitus } \\
\text { symptom } \\
\text { severity }\end{array}$ & - & - & - & - & - & 9 & - & - \\
\hline $\begin{array}{l}\text { Hearing } \\
\text { disabilities }\end{array}$ & - & - & - & - & - & 9 & - & - \\
\hline $\begin{array}{l}\text { Disease-specific } \\
\text { quality of life }\end{array}$ & - & - & - & - & - & 6 & - & - \\
\hline \multicolumn{9}{|c|}{ Life impact core area, 32a: delivery of care-satisfaction / patient preference } \\
\hline Device benefit & - & - & - & - & 2 & 8 & - & - \\
\hline Device use & - & - & 3 & - & 1 & 2 & 1 & 1 \\
\hline Satisfaction & - & - & - & - & 2 & 5 & 1 & - \\
\hline Aversiveness & - & - & - & - & - & 1 & - & \\
\hline \multicolumn{9}{|c|}{ Adverse events core area, 38: adverse events / effects } \\
\hline Adverse effects & - & - & - & 1 & - & 2 & - & 6 \\
\hline
\end{tabular}

evaluating restoring interventions (reported 43 times) rather than rerouting interventions (reported once). The same was true for brain-related assessments of neural activity (restoring studies reported three times; rerouting studies none). In contrast, rerouting studies were also much more concerned about aversiveness (reported 10 times) than were restoring studies (reported once). Furthermore, all dental outcomes were limited to rerouting studies. In fact, all eight reports came from a single study evaluating the SoundBite ${ }^{\mathrm{TM}}$ intraoral device [46-48, 119]. A summary of the split across the interventions is given in Additional file 9.

Overall, restoring intervention studies reported a greater proportion of investigator-administered tests than PROMs, while rerouting intervention studies reported more of a balance of these two instrument types. It was not possible to determine the effect of intervention on the choice of measurement methods because the number of times each method used was generally very small. Perhaps the most striking effect observed was that speech hearing was assessed using the APHAB [140] ease of communication subscale in rerouting studies (reported 13 times) much more often than in restoring studies (reported just once).

\section{Use of measures over time frame}

For both primary and secondary outcomes, there was significant variability in the duration of follow-up period, ranging from acute (baseline) testing to 10 years postintervention. There was notable inconsistency in the 
Table 4 Listing of all unique measurement methods used to assess the most common outcomes in Dodd's taxonomy physiological or clinical \#6, ear and labyrinth category. Only those domains where there was more than one report of the outcome measurement method are selected for reporting here. See Additional file 10 for full details

Measurement methods $(\boldsymbol{n}>\mathbf{1})$ split by outcome domains and type of method
Hearing thresholds, investigator administered
Psychophysical
Pure-tone audiometry
Pure-tone audiometry (bone conduction only)
Soundfield audiometry

$\begin{array}{ll}\text { Primary } & \text { Secondary } \\ \text { outcomes } & \text { outcomes }\end{array}$

Speech in noise, investigator administered

Psychophysical

Bamford-Kowal-Bench Speech in Noise test (BKB-SiN) in four-talker babble, Speech Reception Thresh- 3 olds (SRT)

Bamford-Kowal-Bench Speech in Noise test (BKB-SiN) in multi-talker babble, Speech Reception Thresh- 2 olds (SRT)

Hearing In Noise Test (HINT) in multi-talker babble, Speech Reception Thresholds (SRT)

Hearing in Noise Test (HINT) in R-space restaurant noise, Speech Reception Thresholds (SRT)

Hearing in Noise Test (HINT) noise not specified, Speech Reception Thresholds (SRT)

Hochmair-Schulz-Moser sentence test in speech-shaped noise, Speech Reception Thresholds (SRT)

Leuven Intelligibility Sentences Test (LIST) noise not specified, Speech Reception Thresholds (SRT)

Oldenburg Sentence Test (OISa) noise not specified, Speech Reception Thresholds (SRT)

Quick Speech-In-Noise (QuickSIN) test in multi-talker babble, Speech Reception Thresholds (SRT)

Speech Intelligibility In Noise (SPIN) test in multi-talker babble, percent correct

Speech-in-noise test in speech (not specified), Speech Reception Thresholds (SRT)

\section{peech in noise, PROM}

Multi-item questionnaire

APHAB background noise subscale

\section{Speech in quiet, investigator administered}

Psychophysical

Consonant-Nucleus-Consonant (CNC) word list

Freiburger monosyllabic word discrimination in quiet, Speech Reception Thresholds (SRT)

Monosyllable test (67S test), Japanese version

\section{Speech hearing, PROM}

Multi-item questionnaire

Abbreviated Profile of Hearing Aid Benefit (APHAB) ease of communication subscale

Speech, Spatial and Qualities of Hearing (SSQ) speech subscale

Tinnitus loudness, investigator administered

Psychophysical

Tinnitus Loudness Matching

Tinnitus loudness, PROM

Numerical rating scale

Numerical rating scale (not specified)

Visual analogue scale (not specified)

Spatial hearing, PROM

Multi-item questionnaire

Speech, Spatial and Qualities of Hearing (SSQ) spatial subscale 
Table 4 Listing of all unique measurement methods used to assess the most common outcomes in Dodd's taxonomy physiological or clinical \#6, ear and labyrinth category. Only those domains where there was more than one report of the outcome measurement method are selected for reporting here. See Additional file 10 for full details (Continued)

\begin{tabular}{lll}
\hline Measurement methods $(\boldsymbol{n}>\mathbf{1})$ split by outcome domains and type of method & $\begin{array}{l}\text { Primary } \\
\text { outcomes }\end{array}$ & $\begin{array}{c}\text { Secondary } \\
\text { outcomes }\end{array}$ \\
\hline Spatial Hearing Questionnaire $(\mathrm{SHQ})$, various subscales & - & 8 \\
Localisation, investigator administered & & 1 \\
Psychophysical & 2 & - \\
Horizontal semi-circular array of 7 loudspeakers, angular separation $30^{\circ}$, localisation accuracy & 2 & 1 \\
Horizontal circular array of 5 loudspeakers, angular separation $45^{\circ}$, localisation accuracy & 1 & - \\
Horizontal circular array of 9 out of 33 loudspeakers, angular separation $5.6^{\circ}$, localisation accuracy & 2 & 2 \\
Horizontal circular array of 19 loudspeakers, angular separation $10^{\circ}$, localisation accuracy & 2 & 1 \\
Localisation from one or multiple loudspeakers (not specified) & & \\
\hline
\end{tabular}

number of testing sessions, from a single session to ongoing daily records [50], and when they were conducted after device fitting or surgery. For reporting, time frames were grouped into measures taken in a single session only, at a time point less than 3 months after baseline ('acute'), at a time point from 3 months to less than 1 year after intervention ('early' acclimatisation) and at 1 year or more after intervention ('long'). Eighteen of the 72 included studies were designed as a single session (12 rerouting, 5 restoring, 1 both), 26 had at least one acute follow-up (16 rerouting, 9 restoring, 1 both) mostly at 1 month after baseline, 31 had at least one early followup (11 rerouting, 16 restoring, 4 both) mostly at 6 months after baseline and 26 had at least one long follow-up (6 rerouting, 16 restoring, 4 both).

We evaluated whether there was any change over time in the choice of primary and secondary outcome domains and measurement methods by classifying the data according to the three major Dodd's taxonomy categories [90] (\#6 Ear and labyrinth, \#30 global quality of life and \#32a delivery of care), and according to whether they were investigator-administered tests or PROMs (Fig. 2).

Single-session studies almost exclusively focused on hearing-related outcome domains, but ear and labyrinth accounted for about $50 \%$ of the outcomes assessed, even at the longest time frame. Similarly, single-session studies almost exclusively used investigator-administered testing methods, but over time, a more 50/50 balanced was observed between these and PROMs. This pattern was true for the primary outcomes. However, secondary outcomes were almost always used PROMs, irrespective of the time frame.

\section{Sensitivity analysis}

The final, exploratory objective examined whether we would identify any additional outcomes if we included

Table 5 Listing of all unique measurement methods used to assess the most common outcomes in the Dodd's taxonomy life impact \#30, global quality of life category [90]. Only those domains where there was more than 1 report of the outcome measurement instrument are selected for reporting here. See Additional file 10 for full details

\begin{tabular}{|c|c|c|c|}
\hline \multicolumn{2}{|c|}{ Measurement methods $(n>1)$ split by outcome domains and type of method } & Primary outcomes & Secondary outcomes \\
\hline \multicolumn{4}{|c|}{ Tinnitus-symptom severity, PROM } \\
\hline \multirow[t]{4}{*}{ Multi-item questionnaire } & Tinnitus Handicap Inventory (THI) & 7 & 1 \\
\hline & Tinnitus Questionnaire (TQ) & 2 & 1 \\
\hline & Tinnitus Questionnaire (TQ), German version & 3 & - \\
\hline & Tinnitus Reaction Questionnaire (TRQ) & 3 & - \\
\hline \multicolumn{4}{|l|}{ Hearing disabilities, PROM } \\
\hline Multi-item questionnaire & Speech, Spatial and Qualities of Hearing (SSQ) & 8 & - \\
\hline \multicolumn{4}{|c|}{ Disease-specific quality of life, PROM } \\
\hline \multirow[t]{5}{*}{ Multi-item questionnaire } & Bern Benefit in Single Sided Deafness (BBSS) questionnaire & 1 & 1 \\
\hline & Glasgow Benefit Inventory (GBI) & 3 & 2 \\
\hline & Nijmegen Cochlear Implant Questionnaire (NCIQ) & 1 & 2 \\
\hline & Single Sided Deafness (SSD) questionnaire & 4 & - \\
\hline & Multi-item, multi-domain questionnaire (author's own) & - & 2 \\
\hline
\end{tabular}


Table 6 Listing of all unique measurement methods used to assess the most common outcomes in the Dodd's taxonomy life impact \#32a, Delivery of care (Satisfaction / patient preference) category. Only those domains where there was more than one report of the outcome measurement instrument are selected for reporting here. See Additional file 10 for full details

\begin{tabular}{|c|c|c|c|}
\hline \multicolumn{2}{|c|}{ Measurement methods $(n>1)$ split by outcome domains and type of method } & \multirow[t]{2}{*}{$\begin{array}{l}\text { Primary } \\
\text { outcomes }\end{array}$} & \multirow[t]{2}{*}{$\begin{array}{l}\text { Secondary } \\
\text { outcomes }\end{array}$} \\
\hline \multicolumn{2}{|l|}{ Device benefit, PROM } & & \\
\hline \multirow[t]{3}{*}{ Multi-item questionnaire } & Abbreviated Profile of Hearing Aid Benefit (APHAB) & 6 & - \\
\hline & Glasgow Hearing Aid Benefit Profile (GHABP) & 4 & - \\
\hline & Multi-item, multi-domain Questionnaire (author's own) & 3 & - \\
\hline \multirow[t]{2}{*}{ Numerical rating scale } & $\begin{array}{l}\text { International Outcome Inventory for Hearing Aids (IOI-HA), single item on } \\
\text { benefit }\end{array}$ & 1 & 1 \\
\hline & Visual Analogue Scale (not specified) & 1 & - \\
\hline \multicolumn{4}{|c|}{ Device use, investigator administered } \\
\hline \multirow{2}{*}{$\begin{array}{l}\text { Technical and lab } \\
\text { measures }\end{array}$} & Device log (not specified) & 2 & - \\
\hline & Device log average usage (hrs / day) & 3 & - \\
\hline \multicolumn{4}{|l|}{ Device use, PROM } \\
\hline Multi-item questionnaire & Glasgow Hearing Aid Benefit Profile (GHABP), hearing aid use subscale & 2 & - \\
\hline \multicolumn{4}{|l|}{ Satisfaction, PROM } \\
\hline \multirow[t]{2}{*}{ Multi-item questionnaire } & Glasgow Hearing Aid Benefit Profile (GHABP), various subscales & 1 & 2 \\
\hline & Multi-item, multi-domain questionnaire (author's own) & 2 & - \\
\hline \multicolumn{4}{|l|}{ Aversiveness, PROM } \\
\hline Multi-item questionnaire & Abbreviated Profile of Hearing Aid Benefit (APHAB) aversiveness subscale & 5 & 6 \\
\hline
\end{tabular}

studies where the audiometric eligibility criteria were more lenient than our working definition adopted from the Van de Heyning et al. [1] consensus paper. The outcome domains reported by the 24 additional studies were coded in the same way as described previously. Overall, 205 primary and 80 secondary data items were categorised. None of these reported outcomes had not already been captured by the 72 included studies.

\section{Risk of bias}

Assessment of the 72 included studies focused on (a) whether the outcomes were reported prospectively, and (b) if yes, whether there was consistency between the prospective registration and the published study. Notes on conflicts of interest and study design were also taken.

Although there were 11 clinical trial registrations, only four studies with reported findings had been preregistered. One assessed the SoundBite ${ }^{\mathrm{TM}}$ [46] and three assessed cochlear implantation [120, 121, 131]. The SoundBite $^{\mathrm{TM}}$ study had two discrepancies: adverse effects were not reported in the protocol (NCT01933386) [131] but were reported in the published record discussion, and the pure-tone audiometry (PTA) thresholds range were planned up to $4 \mathrm{kHz}$ in the protocol but reported up to $2 \mathrm{kHz}$ in the study report. All three cochlear implant studies had discrepancies between the measures planned in the registered protocol and those actually reported in the study findings. Other discrepancies included lack of clarity in the report on whether adverse events were assessed at 1 month post-implantation, as per protocol (NCT02259192) [122] and differences between the planned and reported measurement time frames (NCT01749592) [121].

\section{Discussion}

There is a growing general recognition that insufficient attention has been paid to the outcomes measured and reported in clinical trials. The CROSSSD study group has established the need for a core outcome set for SSD interventions and we are the first to identify existing knowledge about outcomes using a systematic review methodology.

\section{Principal findings}

Most studies included in the review evaluated rerouting interventions rather than restoring interventions. There was a large variation in the reported outcome domains, with most studies concentrating on physiological or clinical outcomes, followed by life impact outcomes. Only a small minority of studies reported on resource use and adverse events. Investigators did not always report what their intended outcome domain was, suggesting that their chosen instruments were not actively matched to an outcome domain. With regard to instruments chosen by investigators, a large inconsistency was observed with investigator-administered tests mostly adopted, focusing mainly on speech in noise and spatial-related testing. A diversity within these categories of instruments was also 


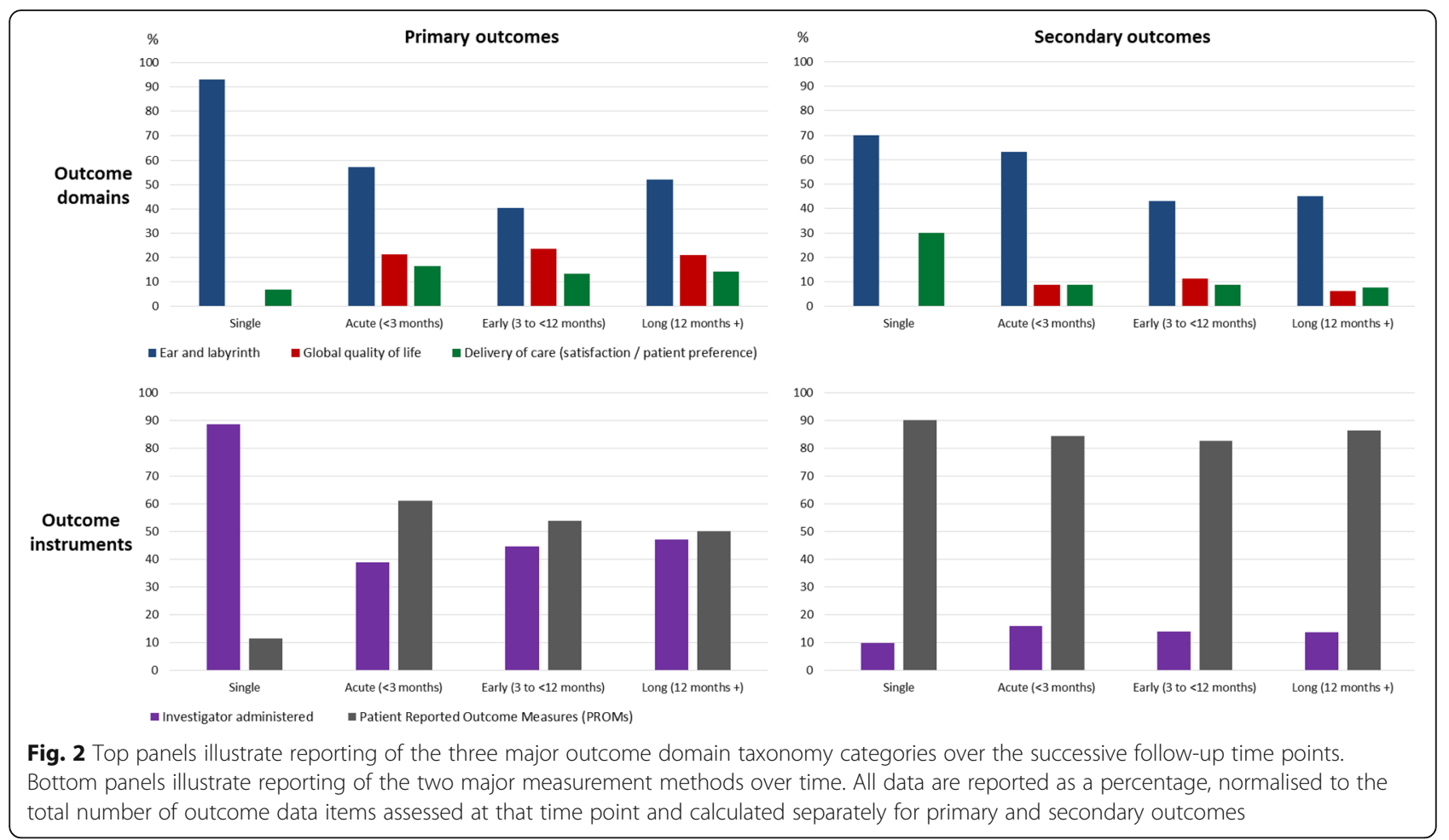

observed with a plethora of signal and noise configurations that do not always fit existing recommendations that aim to reveal both the benefits and drawbacks of hearing devices. Similarly, multi-item questionnaires are frequently utilised but there is no consensus in their selection, nor the intended outcome domains to be measured. Although the range of functional difficulties imposed by SSD, as well as the impact on individual's social and psychological well-being, are well documented [17], similar to other interventions in the hearing field, they are not always assessed in a systematic manner [78, 143, 144]. The time frame when interventions are assessed also varies, so it is challenging to compare the short- and long-term treatment-related changes for rerouting and restoring interventions.

\section{Comparison with other studies}

Our review identifies limitations in the range of reported outcomes in clinical trials that are reflected more broadly across clinical practice in ENT and audiology. In 2016, Van de Heyning led a several expert panel discussions to reach a consensus on a clinical protocol for SSD including a minimum set of outcome measures [1]. This group recommended a core set of three ear and labyrinth and two life impact measures, tested using investigator-administered tests and PROMs, respectively. Ear and labyrinth measures were as follows: (i) hearing thresholds using pure-tone audiometry, (ii) speech in noise perception using a standard audiometric and validated sentence test and a free-field setup in a sound-treated room and (iii) sound localisation using a free-field system with at least seven loudspeakers horizontally distributed with equal angular separation, again in a soundtreated room. In our review, we observed that these were some of the most popular domains reported across the 72 included studies. Common speech in noise materials included the Hearing in Noise Test (HINT) sentences, Oldenburg sentence test (OlSa) and the Speech Intelligibility In Noise (SPIN) test. Measurements for sound localisation perhaps diverged the most from this expert panel recommendation, with numerous studies either using fewer speakers or testing front and back localisation in a circular array. Recommended life impact measures were as follows: (i) quality of life using both disease-specific (speech, spatial, and qualities of hearing (SSQ)) and generic health-related (Health Utilities Index (HUI) Mark 3) questionnaires and (ii) delivery of care using a measure of device use (data logging or patient report). In our review, we actually coded the SSQ questionnaire as an ear and labyrinth assessment (not life impact) because it was most often reported as separate subscale scores for speech hearing, spatial hearing and quality of hearing. We also observed that Health Utilities Index (HUI-3) [145] was rarely reported across the 72 included studies (others were EuroQol-5D-3L [146], WHOQOL-BREF [147] and SF-36 [148]). 
Van de Heyning et al. [1] also recommended tinnitus assessment if applicable, namely tinnitus loudness (using a visual analogue scale (VAS)) and tinnitus symptom severity (using the Tinnitus Functional Index). Our review confirmed that these domains were often limited to restoring interventions (cochlear implant) studies and therefore were perhaps considered less relevant to rerouting interventions for SSD by investigators. It is possible that this recommendation reached consensus because the panel comprised cochlear implant experts attending a cochlear implant conference.

In terms of time frame for outcome measurement, Van de Heyning et al. [1] recommended that outcomes should be collected at baseline, and at 1, 3, 6 and 12 months after intervention. This would mean that all studies should span the acute, early and long-term time points coded in our review. Nevertheless, less than half of the included studies assessed outcome at these time points.

\section{Strengths and limitations of the study}

Our review was guided by good practice as set out in the Core Outcome Measures in Effectiveness Trials (COMET) handbook [77]. In terms of outcome extraction from the academic literature, Williamson et al. [77] state 'it is recommended that all are extracted verbatim from the source manuscript'. This posed some challenges when it came to coding the domains because different investigators used different terminology even when they were probably referring to the same construct. In order to collate the data, our team had to recode some of the domain names, conducting this task in pairs to reduce the impact of individual opinion. For transparency, we provide the authors' original description and our recoding in Additional file 7. This allows external critical review of the CROSSSD core outcome set, whose development will be informed by this review, right back to its inception.

There is currently no consensus on how clinical trial outcomes should be classified [149]. COS researchers often simply agree 'themes' in discussion with advisory groups [150]. However, it is recognised that this lack of a standardised outcome classification system results in inconsistencies due to ambiguity and variation in how outcomes are described across different studies. Recently, a new taxonomy for outcome classification has been developed to promote efficient searching, reporting and classification of trial outcomes [90]. Strengths are that it focuses on general outcomes, is comprehensive, is not disease specific and is applicable to trials for any disease or health condition. Although not part of our published protocol [87], we felt that it was sufficiently important to implement this standard taxonomy in this systematic review. While our findings are presented using this taxonomy, it was insufficient to delineate the different outcome domains with the ear and labyrinth category and so we expanded this category using our own subcategories. Again for transparency, we provide full details of our customised sub-categorisation in Additional file 10.

Also recommended in the COMET handbook [77] is to perform the systematic review in stages to check if outcome saturation is reached. In this sense, our sensitivity analysis can be considered such a check. We had identified 24 additional studies that just missed the eligibility criteria for inclusion, but none of these studies reported any novel outcome domains that had not already been captured in the first stage. As Williamson et al. [77] state 'If there are no further outcomes of importance then the systematic review may be considered complete'.

The longer-term intention of this work is to develop a core outcome set that identifies by consensus a minimum standard for reporting in clinical trials of SSD in adults. This review makes a specific contribution to that endeavour by identifying domains that have been defined in relevant clinical trial designs to date. We recognise that systematic reviews of outcomes simply aggregate the opinions of previous researchers on what outcomes they deemed important to measure. The outcome domains collated in this review will be put forward as potential candidates as outcome domains in a long list that will be considered by a range of stakeholders using a Delphi consensus method [77]. For that long list to be truly comprehensive, it is important to also give participants the option to nominate any new domains that they might consider missing. Here, the patient perspective might be important as they have not yet hitherto been actively involved in COS decision making.

\section{Conclusions}

This review highlights outcome domains and instruments reported by studies that have evaluated rerouting and/or restoring interventions for SSD in adults. The extracted data provides a meticulous catalogue of investigators' chosen outcome domains of which the majority were successfully categorised using the Dodd et al. [90] taxonomy outcome classification system. Our findings emphasise the need to improve trial design and reporting in this area of health research. We hope that guidelines that have been developed explicitly with both rerouting and restoring interventions in mind will have broader take up across the ENT and audiology communities. To improve reporting, we also draw attention to the specialised CONSORT guidelines for reporting harms-related issues in a randomised controlled trial [83]. 


\section{Abbreviations}

APHAB: Abbreviated Profile of Hearing Aid Benefit; BAHA: Bone-anchored hearing aid; BRC: Biomedical Research Centre; CENTRAL: Cochrane Central Register of Controlled Trials; CINAHL: Cumulative Index of Nursing and Allied Health Literature; COMET: Core Outcome Measures in Effectiveness Trials; COS: Core Outcome Set; CROS: Contralateral Routing Of Signals; CROSSSD: Core Rehabilitation Outcome Set for Single Sided Deafness; EMBASE: Excerpta Medica dataBASE; GBI: Glasgow Benefit Inventory; GHABP: Glasgow Hearing Aid Benefit Profile; HINT: Hearing in Noise Test; HUI: Health Utilities Index; ICTRP: International Clinical Trials Registry Platform; IOI-HA: International Outcome Inventory for Hearing Aids; ISRC

TN: International Standard Randomised Controlled Trials Number; MEDL INE: Medical Literature Analysis and Retrieval System Online; MEl: Middle ear implant; NCIQ: Nijmegen Cochlear Implant Questionnaire; NIHR: National Institute for Health Research; OlSa: Oldenburg Sentence Test; PICOS: Population, Intervention, Comparator / Control, Outcomes, Setting: PRISMA: Preferred Reporting Items for Systematic Reviews and MetaAnalyses; PROMs: Patient-reported outcome measures; PROSPERO: International Prospective Register of Systematic Reviews; PTA: Pure-tone audiometry; QuickSIN: Quick Speech-in-Noise; SF-36: Medical Outcome Study Short Form 36; SHQ: Spatial Hearing Questionnaire; SPIN: Speech Intelligibility In Noise; SRT: Speech Reception Thresholds; SSD: Single-sided deafness; SSQ: Speech, Spatial and Qualities Scale; THI: Tinnitus Handicap Inventory; TQ: Tinnitus Questionnaire; USA: United Sates of America; VAS: Visual analogue scale; WHO: World Health Organization; WHOQOL-BREF: World Health Organization Quality of Life Short Form Survey

\section{Supplementary Information}

The online version contains supplementary material available at https://doi. org/10.1186/s13063-021-05160-5.

Additional file 1. PRISMA checklist. PRISMA checklist of items to include when reporting a systematic review.

Additional file 2. Search syntax - MEDLINE and EMBASE. Search syntax for Excerpta Medica dataBASE (EMBASE) and Medical Literature Analysis and Retrieval System Online (MEDLINE) via OvidSP.

Additional file 3. Search syntax - CENTRAL. Search syntax for Cochrane Central Register of Controlled Trials (CENTRAL).

Additional file 4. Title and abstract screening. Methodology for CROSSSD title and abstract article screening.

Additional file 5. Data extraction. CROSSSD data extraction protocol.

Additional file 6. References for all included records. List of records that explicitly fitted the systematic review inclusion criteria as defined by our PROSPERO protocol and were included in the systematic review data extraction $(n=78)$, those that closely fitted and were included for sensitivity analysis only $(n=31)$, and the 12 systematic reviews.

Additional file 7. Data masterfile. An editable version of the data masterfile comprising three worksheets for the 72 studies fitting all criteria, the 24 studies closely fitting the audiometric criteria and the 12 systematic reviews.

Additional file 8. Author queries. Table of records containing missing data that was queried to the corresponding author by email, and outcome.

Additional file 9. Complete list of reported outcome domains. A comprehensive list of all reported primary and secondary outcome domains reported across the 72 included studies, classified according to the Dodd et al. [90] taxonomy. Categories within each core area are arranged by the most frequently used first. The number of individual studies that reported each primary and secondary domain is also listed. $\mathrm{N} / \mathrm{A}=$ none reported.

Additional file 10. Complete list of measurement instruments. A comprehensive list of all primary and secondary measurement instruments $(n=344)$ reported across the 72 included studies, classified according to our domain taxonomy. Where the name of a standard instrument is not given by the authors, a short description is provided instead.

\section{Acknowledgements}

We would like to acknowledge contributions from Nicholas Hogan and Nora Buggy, collaborators with lived experience of SSD and Adele Horobin, Patient and Public Involvement manager whose viewpoints during workshop discussions have guided outcome domains categorisation. Many thanks to Henryk Faas, Assistant Professor in Neuroimaging, Sir Peter Mansfield Imaging Centre, School of Medicine, University of Nottingham who has assisted with the systematic review qualitative synthesis of publications in German. The views expressed in this article are those of the author(s) and not necessarily those of the NHS, the NIHR, or the Department of Health and Social Care.

\section{Authors' contributions}

RK, DAH and PTK managed the review process. RK and PTK conducted the electronic search. RK and DAH verified the data collection for all records. RK, DAH, PTK, CFK, SS and PP conducted the selection process and risk of bias analysis. RK, DAH, CFK, SS, PP and PTK contributed to the data collection. Data analysis, domain grouping and manuscript writing was conducted by RK, DAH and PTK. The final version of the manuscript was read and approved by RK, DAH, CFK, SS, PP and PTK prior to submission.

\section{Funding}

The main body of work for this project is funded by the National Institute for Health Research (NIHR) Nottingham Biomedical Research Centre (BRC), funding reference number BRC-1215-20003. Additional grants obtained: Graham Fraser Foundation Travel Grant to attend the 15th International Conference on Cochlear Implants and other Implantable Auditory Technology (Ci2018.org), where the study was first launched. Oticon Medical ${ }^{\mathrm{TM}}$ provided funding to purchase the DelphiManager software from the COMET Initiative, University of Liverpool. DAH is an NIHR Senior Investigator. The funding bodies have no role in the study design and implementation, writing the report, or decision to submit the report for publication.

\section{Availability of data and materials}

Upon the completion of the study, supporting data will be available upon request.

\section{Declarations}

Ethics approval and consent to participate

This will be sought for each aspect of the study where ethical approval is required. Ethical approval has been authorised by the Nottingham 2 Research Ethics Committee (IRAS Project ID: 239750), Health Research Authority (HRA) and Health and Care Research Wales (HCRW), Reference: 19/ EM/0222.

\section{Consent for publication}

Not applicable.

\section{Competing interests}

PTK declared research grants and support in kind awarded to his institution for research projects in the field of SSD from manufacturers of hearing devices. All other authors declare that they have no competing interests.

\section{Author details}

${ }^{1}$ National Institute for Health Research (NIHR) Nottingham Biomedical Research Centre (BRC), Ropewalk House, 113 The Ropewalk, Nottingham NG1 5DU, UK. ${ }^{2}$ Department of Audiology, Mater Misericordiae University Hospital, Dublin D07 R2WY, Ireland. ${ }^{3}$ Hearing Sciences, Division of Clinical Neuroscience, School of Medicine, University of Nottingham, Nottingham NG7 2UH, UK. "University of Nottingham Malaysia, Jalan Broga, 43500 Semenyih, Selangor Darul Ehsan, Malaysia. ${ }^{5}$ Yorkshire Auditory Implant Service, Bradford Teaching Hospitals Foundation NHS Trust, Bradford Royal Infirmary, Duckworth Lane, Bradford BD9 6RJ, UK. ${ }^{6}$ Department of Otorhinolaryngology, Head and Neck Surgery, Faculty of Medicine, Prince of Songkla University, Songkhla, Thailand. ${ }^{7}$ Nottingham University Hospitals NHS Trust, Queen's Medical Centre, Derby Road, Nottingham NG7 2UH, UK. 
Received: 14 September 2020 Accepted: 27 February 2021 Published online: 20 March 2021

\section{References}

1. Van de Heyning P, Távora-Vieira D, Mertens G, Van Rompaey V, Rajan GP, Müller J, Hempel JM, Leander D, Polterauer D, Marx M, Usami SI, Kitoh R, Miyagawa M, Moteki H, Smilsky K, Baumgartner WD, Keintzel TG, Sprinzl GM, Wolf-Magele A, et al. Towards a unified testing framework for single-sided deafness studies: A consensus paper. Audiol Neurootol. 2017;21:391-8.

2. Everberg G. Etiology of unilateral total deafness studied in a series of children and young adults. Ann Otol Rhinol Laryngol. 1960;69:711-30.

3. Widen JE, Folsom RC, Cone-Wesson B, Carty L, Dunnell JJ, Koebsell K, Levi A, Mancl L, Ohlrich B, Trouba S, Gorga MP, Sininger YS, Vohr BR, Norton SJ. Identification of neonatal hearing impairment: hearing status at 8 to 12 months corrected age using a visual reinforcement audiometry protocol. Ear Hear. 2000;21:471-87.

4. Wu Q, Li X, Sha Y, Dai C. Clinical features and management of Meniere's disease patients with drop attacks. Eur Arch Otorhinolaryngol. 2019;276:66572 .

5. Mirian C, Ovesen T. Intratympanic vs systemic corticosteroids in first-line treatment of idiopathic sudden sensorineural hearing loss: a systematic review and meta-analysis. JAMA Otolaryngol Head Neck Surg. 2020;146:1-8.

6. Chandrasekhar SS, Tsai Do BS, Schwartz SR, Bontempo L, Faucett EA, Finestone SA, Hollingsworth DB, Kelley DM, Kmucha ST, Moonis G, Poling GL, Roberts JK, Stachler RJ, Zeitler DM, Corrigan MD, Nnacheta LC, Satterfield L. Clinical practice guideline: sudden hearing loss (update). Otolaryngol Head Neck Surg. 2019;161:S1-S45

7. Rossini BAA, Penido N d O, Munhoz MSL, Bogaz EA, Curi RS. Sudden sensorioneural hearing loss and autoimmune systemic diseases. Int Arch Otorhinolaryngol. 2017;21:213-23.

8. Staecker H, Nadol JBJ, Ojeman R, Ronner S, McKenna MJ. Hearing preservation in acoustic neuroma surgery: middle fossa versus retrosigmoid approach. Am J Otol. 2000;21:399-404.

9. Douglas SA, Yeung P, Daudia A, Gatehouse S, O'Donoghue GM. Spatial hearing disability after acoustic neuroma removal. Laryngoscope. 2007;117: 1648-51.

10. Daniels RL, Swallow C, Shelton C, Davidson HC, Krejci CS, Harnsberger HR. Causes of unilateral sensorineural hearing loss screened by high-resolution fast spin echo magnetic resonance imaging: review of 1,070 consecutive cases. Am J Otol. 2000;21:173-80.

11. Welsh LW, Welsh JJ, Rosen LF, Dragonette JE. Functional impairments due to unilateral deafness. Ann Otol Rhinol Laryngol. 2004;113:987-93.

12. Snapp HA, Ausili SA. Hearing with one ear: consequences and treatments for profound unilateral hearing loss. J Clin Med. 2020;9:1010.

13. Peters JP, van Zon A, Smit AL, van Zanten GA, de Wit GA, Stegeman I, Grolman W. CINGLE-trial: cochlear implantation for siNGLE-sided deafness, a randomised controlled trial and economic evaluation. BMC Ear Nose Throat Disord. 2015;15:3.

14. Sano H, Okamoto M, Ohhashi K, Iwasaki S, Ogawa K. Quality of life reported by patients with idiopathic sudden sensorineural hearing loss. Otol Neurotol. 2013;34:36-40.

15. Alhanbali S, Dawes P, Lloyd S, Munro KJ. Self-reported listening-related effort and fatigue in hearing-impaired adults. Ear Hear. 2017;38:e39-48.

16. Gatehouse S, Noble W. The speech, spatial and qualities of hearing scale (SSQ). Int J Audiol. 2004;43:85-99.

17. Lucas L, Katiri R, Kitterick PTP. The psychological and social consequences of single-sided deafness in adulthood. Int J Audiol. 2018;57:21-30.

18. Carlsson P-I, Hall M, Lind K-J, Danermark B. Quality of life, psychosocial consequences, and audiological rehabilitation after sudden sensorineural hearing loss. Int J Audiol. 2011;50:139-44.

19. Mohr PE, Feldman JJ, Dunbar JL, McConkey-Robbins A, Niparko JK, Rittenhouse RK, Skinner MW. The societal costs of severe to profound hearing loss in the United States. Int J Technol Assess Health Care. 2000;16: 1120-35.

20. Snapp H. Nonsurgical management of single-sided deafness: contralateral routing of signal. J Neurol Surg B Skull Base. 2019;80:132-8.

21. Snapp HA, Hoffer ME, Liu X, Rajguru SM. Effectiveness in rehabilitation of current wireless CROS technology in experienced bone-anchored implant users. Otol Neurotol. 2017;38:1397-404.

22. Leterme G, Bernardeschi D, Bensemman A, Coudert C, Portal JJ, Ferrary E, Sterkers O, Vicaut E, Frachet B, Grayeli AB. Contralateral routing of signal hearing aid versus transcutaneous bone conduction in single-sided deafness. Audiol Neurotol. 2015;20:251-60.

23. Ryu N-G, Moon IJ, Byun H, Jin SH, Park H, Jang K-S, Cho Y-S. Clinical effectiveness of wireless CROS (Contralateral Routing of Offside Signals) hearing aids. Eur Arch Otorhinolaryngol. 2015;272:2213-9.

24. Snapp HA, Holt FD, Liu X, Rajguru SM. Comparison of speech-in-noise and localization benefits in unilateral hearing loss subjects using contralateral routing of signal hearing aids or bone-anchored implants. Otol Neurotol. 2017;38:11-8.

25. Lin L-M, Bowditch S, Anderson MJ, May B, Cox KM, Niparko JK. Amplification in the rehabilitation of unilateral deafness: speech in noise and directional hearing effects with bone-anchored hearing and contralateral routing of signal amplification. Otol Neurotol. 2006;27:172-82.

26. Choi JE, Ma SM, Park H, Cho YS, Hong SH, Moon J. A comparison between wireless CROS / BiCROS and soft-band BAHA for patients with unilateral hearing loss. PLoS One. 2019;14:e0212503.

27. Harford E, Dodds E. The clinical application of CROS. A hearing aid for unilateral deafness. Arch Otolaryngol. 1966;83:455-64.

28. Grantham DW, Ashmead DH, Haynes DS, Hornsby BWY, Labadie RF, Ricketts TA. Horizontal plane localization in single-sided deaf adults fitted with a bone-anchored hearing aid (Baha). Ear Hear. 2012;33:595-603.

29. Krempaska S, Koval J, Schmid C, Pfiffner F, Kurz A, Kompis M. Influence of directionality and maximal power output on speech understanding with bone anchored hearing implants in single sided deafness. Eur Arch Otorhinolaryngol. 2014;271:1395-400.

30. May BJ, Bowditch S, Liu Y, Eisen M, Niparko JK. Mitigation of informational masking in individuals with single-sided deafness by integrated bone conduction hearing aids. Ear Hear. 2014;35:41-8.

31. Niparko JK, Cox KM, Lustig LR. Comparison of the bone anchored hearing aid implantable hearing device with contralateral routing of offside signal amplification in the rehabilitation of unilateral deafness. Otol Neurotol. 2003;24:73-8.

32. Wazen JJ, Spitzer JB, Ghossaini SN, Fayad JN, Niparko JK, Cox K, Brackmann DE, Soli SD. Transcranial contralateral cochlear stimulation in unilateral deafness. Otolaryngol Head Neck Surg. 2003;129:248-54.

33. Pfiffner F, Kompis M, Flynn M, Asnes K, Arnold A, Stieger C. Benefits of lowfrequency attenuation of Baha ${ }^{\oplus}$ in single-sided sensorineural deafness. Ear Hear. 2011;32:40-5.

34. Moore BCJ, Popelka GR. Preliminary comparison of bone-anchored hearing instruments and a dental device as treatments for unilateral hearing loss. Int J Audiol. 2013;52:678-86.

35. Pai I, Kelleher C, Nunn T, Pathak N, Jindal M, O'Connor AF, Jiang D. Outcome of bone-anchored hearing aids for single-sided deafness: a prospective study. Acta Otolaryngol. 2012;132:751-5.

36. Oeding K, Valente M, Kerckhoff J. Effectiveness of the directional microphone in the Baha ${ }^{\oplus}$ Divino ${ }^{\mathrm{TM}}$. J Am Acad Audiol. 2010;21:546-57.

37. Oeding K, Valente M. The effectiveness of the directional microphone in the Oticon Medical Ponto Pro in participants with unilateral sensorineural hearing loss. J Am Acad Audiol. 2013;24:701-13.

38. Weber BA, Roush J, McElveen JTJ. Application of an implantable bone conduction hearing device to patients with unilateral sensorineural hearing loss. Laryngoscope. 1992;102:538-42.

39. Bosman AJ, Hol MKS, Snik AFM, Mylanus EAM, Cremers CWRJ. Boneanchored hearing aids in unilateral inner ear deafness. Acta Otolaryngol. 2003;123:258-60.

40. Gluth MB, Eager KM, Eikelboom RH, Atlas MD. Long-term benefit perception, complications, and device malfunction rate of bone-anchored hearing aid implantation for profound unilateral sensorineural hearing loss. Otol Neurotol. 2010;31:1427-34.

41. Linstrom CJ, Silverman CA, Yu G-PP. Efficacy of the bone-anchored hearing aid for single-sided deafness. Laryngoscope. 2009;119:713-20.

42. Newman CW, Sandridge SA, Wodzisz LM. Longitudinal benefit from and satisfaction with the Baha system for patients with acquired unilateral sensorineural hearing loss. Otol Neurotol. 2008;29:1123-31.

43. Agterberg MJH, Snik AFM, Van de Goor RMG, Hol MKS, Van Opstal AJ. Sound-localization performance of patients with single-sided deafness is not improved when listening with a bone-conduction device. Hear Res. 2019;372:62-8.

44. Mertens G, Gilles A, Bouzegta R, Van De Heyning P. A prospective randomized crossover study in single sided deafness on the new noninvasive adhesive bone conduction hearing system. Otol Neurotol. 2018;39: 940-9. 
45. Moteki H, Kitoh R, Usami SI. The availability of an adhesive bone conduction hearing device: a preliminary report of a single-center experience. Acta Otolaryngol. 2020;140:319-26.

46. Miller R, Hujoel P, Murray M, Popelka GR. Safety of an intra-oral hearing device utilizing a split-mouth research design. J Clin Dent. 2011;22:159-62.

47. Murray M, Miller R, Hujoel P, Popelka GR. Long-term safety and benefit of a new intraoral device for single-sided deafness. Otol Neurotol. 2011;32:1262-9.

48. Popelka GR, Derebery J, Blevins NH, Murray M, Moore BCJ, Sweetow RW, Wu $B$, Katsis M. Preliminary evaluation of a novel bone-conduction device for single-sided deafness. Otol Neurotol. 2010;31:492-7.

49. Gurgel RK, Shelton C. The SoundBite hearing system: patient-assessed safety and benefit study. Laryngoscope. 2013;123:2807-12.

50. Schmerber S, Deguine O, Marx M, van de Heyning P, Sterkers O, Mosnier I, Garin P, Godey B, Vincent C, Venail F, Mondain M, Deveze A, Lavieille JP, Karkas A. Safety and effectiveness of the Bonebridge transcutaneous active direct-drive bone-conduction hearing implant at 1-year device use. Eur Arch Otorhinolaryngol. 2017;274:1835-51.

51. Laske RD, Röösli C, Pfiffner F, Veraguth D, Huber AM. Functional results and subjective benefit of a transcutaneous bone conduction device in patients with single-sided deafness. Otol Neurotol. 2015;36:1151-6.

52. Arndt S, Laszig R, Aschendorff A, Beck R, Schild C, Hassepass F, Ihorst G, Kroeger S, Kirchem P, Wesarg T. Unilateral deafness and cochlear implantation: audiological diagnostic evaluation and outcomes. HNO. 2011; 59:437-46.

53. Härkönen K, Kivekas I, Rautiainen M, Kotti V, Sivonen V, Vasama J-P. Singlesided deafness: the effect of cochlear implantation on quality of life, quality of hearing, and working performance. ORL J Otorhinolaryngol Relat Spec. 2015;77:339-45.

54. Härkönen K, Kivekas I, Kotti V, Sivonen V, Vasama J-P. Hybrid cochlear implantation: quality of life, quality of hearing, and working performance compared to patients with conventional unilateral or bilateral cochlear implantation. Eur Arch Otorhinolaryngol. 2017;274:3599-604.

55. Grossmann W, Brill S, Moeltner A, Mlynski R, Hagen R, Radeloff A. Cochlear implantation improves spatial release from masking and restores localization abilities in single-sided deaf patients. Otol Neurotol. 2016;37:658-64.

56. Távora-Vieira D, Boisvert I, McMahon CM, Maric V, Rajan GP. Successful outcomes of cochlear implantation in long-term unilateral deafness: brain plasticity? Neuroreport. 2013;24:724-9.

57. Távora-Vieira D, De Ceulaer G, Govaerts PJ, Rajan GP. Cochlear implantation improves localization ability in patients with unilateral deafness. Ear Hear. 2015;36:e93-8.

58. Távora-Vieira D, Marino R, Acharya A, Rajan GP. The impact of cochlear implantation on speech understanding, subjective hearing performance, and tinnitus perception in patients with unilateral severe to profound hearing loss. Otol Neurotol. 2015;36:430-6.

59. Arndt S, Aschendorff A, Laszig R, Beck R, Schild C, Kroeger S, Ihorst G, Wesarg T. Comparison of pseudobinaural hearing to real binaural hearing rehabilitation after cochlear implantation in patients with unilateral deafness and tinnitus. Otol Neurotol. 2011;32:39-47.

60. Arndt S, Laszig R, Aschendorff A, Hassepass F, Beck R, Wesarg T. Cochlear implant treatment of patients with single-sided deafness or asymmetric hearing loss German version. HNO. 2017;65:586-98.

61. Doge J, Baumann U, Weissgerber T, Rader T. Single-sided deafness: impact of cochlear implantation on speech perception in complex noise and on auditory localization accuracy. Otol Neurotol. 2017;38:e563-9.

62. Jacob R, Stelzig Y, Nopp P, Schleich P. Audiological results with cochlear implants for single-sided deafness. HNO. 2011;59:453-60.

63. Firszt JB, Holden LK, Reeder RM, Waltzman SB, Arndt S. Auditory abilities after cochlear implantation in adults with unilateral deafness: a pilot study. Otol Neurotol. 2012;33:1339-46.

64. Legris E, Galvin J, Roux S, Gomot M, Aoustin JM, Marx M, He S, Bakhos D. Cortical reorganization after cochlear implantation for adults with singlesided deafness. PLoS One. 2018;13:e0204402.

65. Galvin JJ, Fu QJ, Wilkinson EP, Mills D, Hagan SC, Lupo JE, Padilla M, Shannon RV. Benefits of cochlear implantation for single-sided deafness: data from the House Clinic-University of Southern California-University of California, Los Angeles Clinical Trial. Ear Hear. 2019;40:766-81.

66. Peter N, Kleinjung T, Probst R, Hemsley C, Veraguth D, Huber A, Caversaccio M, Kompis M, Mantokoudis G, Senn P, Wimmer W. Cochlear implants in single-sided deafness - clinical results of a Swiss multicentre study. Swiss Med Wkly. 2019;149:w20171.
67. Poncet-Wallet C, Mamelle E, Godey B, Truy E, Guevara N, Ardoint M, Gnansia D, Hoen M, Saaï S, Mosnier I, Lescanne E, Bakhos D, Vincent C. Prospective multicentric follow-up study of cochlear implantation in adults with singlesided deafness: tinnitus and audiological outcomes. Otol Neurotol. 2020;41: 458-66.

68. Lorens A, Kruszyńska M, Obrycka A, Skarzynski PH, Wilson B, Skarzynski H. Binaural advantages in using a cochlear implant for adults with profound unilateral hearing loss. Acta Otolaryngol. 2019;139:153-61.

69. Marx M, Costa NN, Lepage B, Taoui S, Molinier L, Deguine O, Fraysse B. Cochlear implantation as a treatment for single-sided deafness and asymmetric hearing loss: a randomized controlled evaluation of cost-utility. BMC Ear Nose Throat Disord. 2019;19:1.

70. Litovsky RY, Moua K, Godar S, Kan A, Misurelli SM, Lee DJ. Restoration of spatial hearing in adult cochlear implant users with single-sided deafness. Hear Res. 2019;372:69-79.

71. Häußler SM, Köpke V, Knopke S, Gräbel S, Olze H. Multifactorial positive influence of cochlear implantation on patients with single-sided deafness. Laryngoscope. 2020;130:500-6.

72. Kitoh R, Moteki H, Nishio S, Shinden S, Kanzaki S, Iwasaki S, Ogawa K, Usami SI. The effects of cochlear implantation in Japanese single-sided deafness patients: five case reports. Acta Otolaryngol. 2016;136:460-4.

73. Ahmed MF, Khater A. Tinnitus suppression after cochlear implantation in patients with single-sided deafness. Egypt J Otolaryngol. 2017;33:61.

74. Gargon E, Gurung B, Medley N, Altman DG, Blazeby JM, Clarke M, Williamson PR. Choosing important health outcomes for comparative effectiveness research: a systematic review. PLoS One. 2014;9:e99111.

75. Chalmers I, Glasziou P. Avoidable waste in the production and reporting of research evidence. Lancet. 2009;374:86-9.

76. Kitterick PT, Smith SN, Lucas L. Hearing instruments for unilateral severe-toprofound sensorineural hearing loss in adults: a systematic review and meta-analysis. Ear Hear. 2016;37:495-507.

77. Williamson PR, Altman DG, Bagley H, Barnes KL, Blazeby JM, Brookes ST, Clarke M, Gargon E, Gorst S, Harman N, Kirkham JJ, McNair A, Prinsen CAC, Schmitt J, Terwee CB, Young B. The COMET Handbook: Version 1.0. Trials. 2017;18:1-50.

78. Hall DA, Kitterick P, Heffernan E, Fackrell K, Lucas L, Ferguson M. How do we know that our patients have benefitted from our ENT / audiological interventions? Presented at the Annual Meeting of ADANO 2016 in Berlin. Otol Neurotol. 2019;40:e474-81.

79. Clarke M. Standardising outcomes for clinical trials and systematic reviews. Trials. 2007:8:39.

80. Sinha IP, Williamson PR, Smyth RL. Outcomes in clinical trials of inhaled corticosteroids for children with asthma are narrowly focussed on short term disease activity. PLoS One. 2009;4:e6276.

81. Gandhi GY, Murad MH, Fujiyoshi A, Mullan RJ, Flynn DN, Elamin MB, Swiglo BA, Isley WL, Guyatt GH, Montori VM. Patient-important outcomes in registered diabetes trials. JAMA. 2008;299:2543-9.

82. Williamson P, Clarke M. The COMET (Core Outcome Measures in Effectiveness Trials) initiative: its role in improving Cochrane reviews. Cochrane Database Syst Rev. 2012;13:ED000041.

83. Moher D, Hopewell S, Schulz KF, Montori V, Gøtzsche PC, Devereaux PJ, Elbourne D, Egger M, Altman DG, Gotzsche PC, Devereaux PJ, Elbourne D, Egger M, Altman DG. CONSORT 2010 explanation and elaboration: updated guidelines for reporting parallel group randomised trials. BMJ. 2010;340: c869.

84. Chan A-W, Tetzlaff JM, Gotzsche PC, Altman DG, Mann H, Berlin JA, Dickersin K, Hrobjartsson A, Schulz KF, Parulekar WR, Krleza-Jeric K, Laupacis A, Moher D. SPIRIT 2013 explanation and elaboration: guidance for protocols of clinical trials. BMJ. 2013;346:e7586.

85. Zwarenstein M, Treweek S, Gagnier JJ, Altman DG, Tunis S, Haynes B, Oxman AD, Moher D. Improving the reporting of pragmatic trials: an extension of the CONSORT statement. BMJ. 2008;337:a2390.

86. Katiri R, Hall DA, Buggy N, Hogan N, Horobin A, van de Heyning P, Firszt JB, Bruce IA, Kitterick PT. Core Rehabilitation Outcome Set for Single Sided Deafness (CROSSSD) study: protocol for an international consensus on outcome measures for single sided deafness interventions using a modified Delphi survey. Trials. 2020;21:238.

87. Hall DA, Kitterick PT, Katiri R. Systematic review of outcome domains and instruments used in designs of clinical trials for interventions that seek to restore bilateral and binaural hearing in adults with unilateral sensorineural severe to profound hearing loss ('Single Sided Deafness'). In: PROSPERO; 
2018. https://www.crd.york.ac.uk/prospero/display_record.php?RecordID= 84274. Accessed 10 Aug 2020.

88. Liberati A, Altman DG, Tetzlaff J, Mulrow C, Gotzsche PC, loannidis JPA, Clarke M, Devereaux PJ, Kleijnen J, Moher D. The PRISMA statement for reporting systematic reviews and meta-analyses of studies that evaluate health care interventions: explanation and elaboration. J Clin Epidemiol. 2009;62:e1-34.

89. Bovo R, Prosser S, Ortore RP, Martini A. Speech recognition with BAHA simulator in subjects with acquired unilateral sensorineural hearing loss. Acta Otolaryngol. 2011;131:633-9.

90. Dodd S, Clarke M, Becker L, Mavergames C, Fish R, Williamson PR. A taxonomy has been developed for outcomes in medical research to help improve knowledge discovery. J Clin Epidemiol. 2018;96:84-92.

91. Moher D, Liberati A, Tetzlaff J, Altman DG. Preferred reporting items for systematic reviews and meta-analyses: the PRISMA statement. BMJ. 2009; 339:b2535.

92. Sprinzl GM, Wolf-Magele A. The Bonebridge bone conduction hearing implant: indication criteria, surgery and a systematic review of the literature. Clin Otolaryngol. 2016;41:131-43.

93. Blasco MA, Redleaf MI. Cochlear implantation in unilateral sudden deafness improves tinnitus and speech comprehension: meta-analysis and systematic review. Otol Neurotol. 2014;35:1426-32.

94. van Zon A, Peters JPM, Stegeman I, Smit AL, Grolman W. Cochlear implantation for patients with single-sided deafness or asymmetrical hearing loss: a systematic review of the evidence. Otol Neurotol. 2015;36:209-19.

95. Peters JPM, Smit AL, Stegeman I, Grolman W. Review: bone conduction devices and contralateral routing of sound systems in single-sided deafness. Laryngoscope. 2015;125:218-26.

96. Kitterick PT, Lucas L, Smith SN. Improving health-related quality of life in single-sided deafness: A systematic review and meta-analysis. Audiol Neurotol. 2015;20:79-86.

97. Kim G, Ju HM, Lee SH, Kim H-S, Kwon JA, Seo YJ. Efficacy of bone-anchored hearing aids in single-sided deafness: a systematic review. Otol Neurotol. 2017;38:473-83.

98. Wendrich AW, Kroese TE, Peters JPM, Cattani G, Grolman W. Systematic review on the trial period for bone conduction devices in single-sided deafness: rates and reasons for rejection. Otol Neurotol. 2017;38:632-41.

99. Cabral Junior F, Hausen Pinna M, Dourado Alves R, dos Santos Malerbi AF, Ferreira Bento R. Cochlear implantation and single-sided deafness: A systematic review of the literature. Int Arch Otorhinolaryngol. 2016;20:69-75.

100. Peter N, Liyanage N, Pfiffner F, Huber A, Kleinjung T. The influence of cochlear implantation on tinnitus in patients with single-sided deafness: a systematic review. Otolaryngol Head Neck Surg. 2019;161:576-88.

101. Magele A, Schoerg P, Stanek B, Gradl B, Sprinzl GM. Active transcutaneous bone conduction hearing implants: systematic review and meta-analysis. PLoS One. 2019;14:e0221484.

102. Cohen SM, Svirsky MA. Duration of unilateral auditory deprivation is associated with reduced speech perception after cochlear implantation: a single-sided deafness study. Cochlear Implants Int. 2019;20:51-6.

103. Canis M, Ihler F, Blum J, Matthias C. CT-assisted navigation for retrosigmoidal implantation of the Bonebridge. HNO. 2013;61:1038-44.

104. Cho B, Matsumoto N, Mori M, Komune S, Hashizume M. Image-guided placement of the Bonebridge without surgical navigation equipment. Int $J$ Comput Assist Radiol Surg. 2014;9:845-55.

105. Güldner C, Heinrichs J, Weiß R, Zimmermann AP, Dassinger B, Bien S, Werner JA, Diogo I. Visualisation of the Bonebridge by means of $\mathrm{CT}$ and CBCT. Eur J Med Res. 2013;18:30.

106. Huber AM, Sim JH, Xie YZ, Chatzimichalis M, Ullrich O, Röösli C. The Bonebridge: preclinical evaluation of a new transcutaneously-activated bone anchored hearing device. Hear Res. 2013;301:93-9.

107. Nospes S, Mann W, Keilmann A. Magnetic resonance imaging in patients with magnetic hearing implants: overview and procedural management. Radiologe. 2013;53:1026-32.

108. Finke $M$, Bonitz $H$, Lyxell B, Illg A. Cochlear implant effectiveness in postlingual single-sided deaf individuals: what's the point? Int J Audiol. 2017:56:417-23.

109. Wimmer W, Gerber N, Guignard J, Dubach P, Kompis M, Weber S, Caversaccio M. Topographic bone thickness maps for Bonebridge implantations. Eur Arch Otorhinolaryngol. 2015;272:1651-8.

110. Barbara M, Perotti M, Gioia B, Volpini L, Monini S. Transcutaneous boneconduction hearing device: audiological and surgical aspects in a first series of patients with mixed hearing loss. Acta Otolaryngol. 2013;133:1058-64.
111. Ihler F, Volbers L, Blum J, Matthias C, Canis M. Preliminary functional results and quality of life after implantation of a new bone conduction hearing device in patients with conductive and mixed hearing loss. Otol Neurotol. 2014:35:211-5.

112. Lassaletta L, Sanchez-Cuadrado I, Muñoz E, Gavilan J. Retrosigmoid implantation of an active bone conduction stimulator in a patient with chronic otitis media. Auris Nasus Larynx. 2014;41:84-7.

113. Mertens G, Desmet J, Snik AFM, Van de Heyning P. An experimental objective method to determine maximum output and dynamic range of an active bone conduction implant: the Bonebridge. Otol Neurotol. 2014;35: $1126-30$.

114. Schnabl J, Wolf-Magele A, Pok SM, Schoerg P, Hirtler L, Schloegel M, Sprinzl G. Intraoperative measurement for a new transcutaneous bone conduction hearing implant. Otol Neurotol. 2014;35:1242-7.

115. Sprinzl G, Lenarz T, Ernst A, Hagen R, Wolf-Magele A, Mojallal H, Todt I, Mlynski R, Wolframm MD. First European multicenter results with a new transcutaneous bone conduction hearing implant system: short-term safety and efficacy. Otol Neurotol. 2013;34:1076-83.

116. Lassaletta L, Calvino M, Zernotti M, Gavilan J. Postoperative pain in patients undergoing a transcutaneous active bone conduction implant (Bonebridge) Eur Arch Otorhinolaryngol. 2016;273:4103-10.

117. Clarós P, Diouf MS, Clarós A. Setting up a "Bonebridge". Rev Laryngol Otol Rhinol. 2012;133:217-20.

118. de Wolf MJF, Hol MKS, Huygen PLM, Mylanus EAM, Cremers CWRJ. Nijmegen results with application of a bone-anchored hearing aid in children: simplified surgical technique. Ann Otol Rhinol Laryngol. 2008;117:805-14.

119. Popelka GR. SoundBite hearing system by Sonitus medical: a new approach to single-sided deafness. Semin Hear. 2010;31:393-409.

120. Marx M. Cochlear implantation in single sided deafness and asymmetrical hearing loss: a cost-utility study: ClinicalTrials.gov; 2014. https://clinicaltrials. gov/ct2/show/NCT02204618. Accessed 10 Aug 2020

121. Kleinjung T. Single-sided deafness and cochlear implants: ClinicalTrials.gov; 2012. https:/clinicaltrials.gov/ct2/show/NCT01749592. Accessed 10 Aug 2020

122. Shannon RV. Cochlear implants for adults with single-sided deafness: ClinicalTrials.gov; 2014. https://clinicaltrials.gov/ct2/show/NCT02259192. Accessed 10 Aug 2020

123. Gnansia D, Frachet B. Tinnitus treatment with cochlear implant in single sided deafness: ClinicalTrials.gov; 2016. https://clinicaltrials.gov/ct2/show/ NCT02966366. Accessed 10 Aug 2020

124. Song J-J, Punte AK, De Ridder D, Vanneste S, Van de Heyning P. Neural substrates predicting improvement of tinnitus after cochlear implantation in patients with single-sided deafness. Hear Res. 2013;299:1-9.

125. Brendel M, Hamacher V. Influence of Contralateral Routing of Signals (CROS) on hearing abilities of different groups of cochlear implant user: drks.de; 2018. http://www.drks.de/DRKS00013973. Accessed 10 Aug 2020

126. Willeboer K. Parametric cochlear implant map adjustment by implant recipients: TrialRegister.nl; 2005. https://www.trialregister.nl/trial/452. Accessed 10 Aug 2020

127. Smith MD, Knappett R. Hearing handicap in patients with single sided deafness: ClinicalTrials.gov; 2015. https://clinicaltrials.gov/ct2/show/NCT0252 5640. Accessed 10 Aug 2020

128. Doobe G, Ernst A, Ramalingam R, Mittmann P, Todt I. Simultaneous labyrinthectomy and cochlear implantation for patients with single-sided Ménière's disease and profound sensorineural hearing loss. Biomed Res Int. 2015;2015:457318.

129. Kubo T, Yamamoto KI, Iwaki T, Doi K, Tamura M. Different forms of dizziness occurring after cochlear implant. Eur Arch Otorhinolaryngol. 2001;258:9-12.

130. Hill SL 3rd, Marcus A, Digges ENB, Gillman N, Silverstein H. Assessment of patient satisfaction with various configurations of digital CROS and BiCROS hearing aids. Ear Nose Throat J. 2006;85:427-30.

131. Syms C, Galow L. Evaluation of benefit for treatment of single sided deafness (SSD) between two bone conduction prosthetic devices; osseointegrated implant versus maxilla anchored removable oral appliance ('SoundBite'): ClinicalTrials.gov; 2013. https://clinicaltrials.gov/ct2/show/ NCT01933386. Accessed 10 Aug 2020

132. Buechner A, Brendel M, Lesinski-Schiedat A, Wenzel G, Frohne-Buechner C, Jaeger B, Lenarz T. Cochlear implantation in unilateral deaf subjects associated with ipsilateral tinnitus. Otol Neurotol. 2010;31:1381-5.

133. Dillon MT, Buss E, Anderson ML, King ER, Deres EJ, Buchman CA, Brown KD, Pillsbury HC. Cochlear implantation in cases of unilateral hearing loss: initial localization abilities. Ear Hear. 2017;38:611-9. 
134. Dillon MT, Buss E, Rooth MA, King ER, Deres EJ, Buchman CA, Pillsbury HC, Brown KD. Effect of cochlear implantation on quality of life in adults with unilateral hearing loss. Audiol Neurotol. 2017;22:259-71.

135. Hol MKS, Kunst SJW, Snik AFM, Cremers CWRJ. Pilot study on the effectiveness of the conventional CROS, the transcranial CROS and the BAHA transcranial CROS in adults with unilateral inner ear deafness. Eur Arch Otorhinolaryngol. 2010;267:889-96.

136. Louza J, Hempel JM, Krause E, Berghaus A, Müller J, Braun T. Patient benefit from cochlear implantation in single-sided deafness: a 1-year follow-up. Eur Arch Otorhinolaryngol. 2017;274:2405-9.

137. Sladen DP, Carlson ML, Dowling BP, Olund AP, Teece K, DeJong MD, Breneman A, Peterson A, Beatty CW, Neff BA, Driscoll CL. Early outcomes after cochlear implantation for adults and children with unilateral hearing loss. Laryngoscope. 2017;127:1683-8.

138. Dumon T, Medina M, Sperling NM. Punch and drill: implantation of bone anchored hearing device through a minimal skin punch incision versus implantation with dermatome and soft tissue reduction. Ann Otol Rhinol Laryngol. 2016;125:199-206.

139. Gatehouse S. A self-report outcome measure for the evaluation of hearing aid fittings and services. Health Bull (Raleigh). 1999;57:424-36.

140. Cox RM, Alexander GC. The abbreviated profile of hearing aid benefit. Ear Hear. 1995;16:176-86.

141. Nilsson M, Soli SD, Sullivan JA. Development of the hearing in noise test for the measurement of speech reception thresholds in quiet and in noise. J Acoust Soc Am. 1994;95:1085-99.

142. Newman CW, Jacobson GP, Spitzer JB. Development of the tinnitus handicap inventory. Arch Otolaryngol Head Neck Surg. 1996;122:143-8.

143. Hall DA, Haider H, Szczepek AJ, Lau P, Rabau S, Jones-Diette J, Londero A, Edvall NK, Cederroth CR, Mielczarek M, Fuller T, Batuecas-Caletrio A, Brueggemen P, Thompson DM, Norena A, Cima RFF, Mehta RL, Mazurek B. Systematic review of outcome domains and instruments used in clinical trials of tinnitus treatments in adults. Trials. 2016;17:270

144. Danermark B, Granberg S, Kramer SE, Selb M, Möller C. The creation of a comprehensive and a brief core set for hearing loss using the international classification of functioning, disability and health. Am J Audiol. 2013;22:323-8.

145. Horsman J, Furlong W, Feeny D, Torrance G. The Health Utilities Index (HUI): concepts, measurement properties and applications. Heal Qual Life Outcomes. 2003;1:54

146. EuroQol Group. EuroQol - a new facility for the measurement of healthrelated quality of life. Health Policy (New York). 1990;16:199-208.

147. Skevington SM, Lotfy M, O'Connell KA. The World Health Organization's WHOQOL-BREF quality of life assessment: psychometric properties and results of the international field trial. A report from the WHOQOL group. Qual Life Res. 2004;13:299-310.

148. Ware JEJ, Sherbourne CD. The MOS 36-item short-form health survey (SF36). I. Conceptual framework and item selection. Med Care. 1992:30:473-83.

149. Gorst SL, Gargon E, Clarke M, Smith V, Williamson PR. Choosing important health outcomes for comparative effectiveness research: an updated review and identification of gaps. PLoS One. 2016;11:e0168403.

150. Alkhaffaf B, Glenny AM, Blazeby JM, Williamson P, Bruce IA. Standardising the reporting of outcomes in gastric cancer surgery trials: protocol for the development of a core outcome set and accompanying outcome measurement instrument set (the GASTROS study). Trials. 2017;18:370.

\section{Publisher's Note}

Springer Nature remains neutral with regard to jurisdictional claims in published maps and institutional affiliations.

Ready to submit your research? Choose BMC and benefit from:
- fast, convenient online submission
- thorough peer review by experienced researchers in your field
- rapid publication on acceptance
- support for research data, including large and complex data types
- gold Open Access which fosters wider collaboration and increased citations
- maximum visibility for your research: over 100M website views per year
At BMC, research is always in progress.
Learn more biomedcentral.com/submissions

